\title{
Taxonomic descriptions of nine new species of the goblin spider genera Cavisternum, Grymeus, Ischnothyreus, Opopaea, Pelicinus and Silhouettella (Araneae, Oonopidae) from Sri Lanka
}

\author{
U.G.S.L. Ranasinghe ${ }^{1}$, Suresh P. Benjamin ${ }^{1}$ \\ 1 National Institute of Fundamental Studies, Hantana Road, Kandy, Sri Lanka \\ http://zoobank.org/ACAEC71D-964C-4314-AAA7-A404F23A6569 \\ Corresponding author: Suresh P. Benjamin (suresh.benjamin@gmail.com)
}

Received 22 March 2018

Accepted 22 May 2018

Published 21 June 2018

Academic editor:

Danilo Harms

Key Words

Biodiversity

Ceylon

leaf litter

systematics

\begin{abstract}
Nine new species of goblin spiders are described in six different genera: Cavisternum bom n. sp., Grymeus dharmapriyai n. sp., Ischnothyreus chippy n. sp., Opopaea spinosiscorona n. sp., Pelicinus snooky n. sp., P. tumpy n. sp., Silhouettella saaristoi n. sp., S. snippy n. sp. and S. tiggy n. sp. Three genera are recorded for the first time in Sri Lanka: Cavisternum, Grymeus and Silhouettella. The first two genera are reported for the first time outside of Australia. Sri Lankan goblin spider diversity now comprises 45 described species in 13 different genera.
\end{abstract}

\section{Introduction}

Sri Lanka is home to 393 species of spiders classified in 45 families (World Spider Catalog 2018). A large proportion of these species was described over the past two decades (Azarkina 2004; Baehr and Ubick 2010; Bayer 2012; Benjamin 2000, 2001, 2004, 2006, 2010, 2015; Benjamin and Jocqué 2000; Benjamin and Kanesharatnam 2016; Dong et al. 2016; Dunlop and Jekel 2009; Eichenberger et al., 2012; Grismado et al. 2011; Huber 2005, 2011; Huber and Benjamin 2005; Jäger 2003; Kanesharatnam and Benjamin 2016; Kim et al. 2013, 2014; Nanayakkara et al. 2012; Platnick et al. 2011; Polotow and Griswold 2017; Ranasinghe and Benjamin 2016a, b, c; Smith 2004) and almost all of the new species (55 of 58 new species) are endemics that are presently not known from anywhere else outside of Sri Lanka. Taxonomic studies are necessary to document biodiversity and provide base data for effective nature conservation (Alvarez-Padilla et al. 2015). Field work conducted by us during last few years have shown the presence of an abundant, largely unexplored spider fauna living in the forest patches of the island (Ranasinghe and Benjamin 2016a, b, c, 2018; Kanesharatnam and Benjamin 2016; Benjamin and Kanesharatnam 2016, in press; Batuwita and Benjamin 2014). This now concluded project on Sri Lankan Oonopidae was initiated to discover new species (Ranasinghe and Benjamin 2016a, b, c, 2018; Ranasinghe 2017) and as a result of this project, 19 new species were discovered from forest habitats across the island (Ranasinghe and Benjamin 2016a, b, c, 2018). Of the more than 100 localities sampled in Sri Lanka, many goblin spider species were often only found in a few sites whilst others were found only in a single forest patch and absent even in the immediate surrounding forests. These species, short-range endemics with very restricted distributions, may prove to be important flagship taxa for monitoring the effects of climate change and other threats on forest habitats in Sri Lanka (Baehr 2011). This paper reports the discovery of nine new species of goblin spiders of the genera, Cavisternum, Grymeus, Ischnothyreus, Opopaea, Pelicinus and Silhouettella. 


\section{Materials and methods}

Specimens were collected by sifting litter and leaving the residue overnight in a Winkler extractor or by hand-sorting the residue. The collected specimens were examined using an Olympus SZX7 stereomicroscope. Specimens were preserved in $70 \%$ ethanol. Preserved specimens were identified using recently published studies (Álvarez-Padilla et al. 2015; Baehr et al. 2010, 2013a, b; Harvey and Baehr 2013; Harvey 1987; Ott and Harvey 2008; Platnick et al. 2011, 2012; Saaristo 2007). Specimen examination: Male palps (left) were dissected and immersed in Kaiser's glycerol gelatin (Merck KGaA, Darmstadt, Germany), slide mounted, observed and illustrated with the aid of an Olympus BX51 compound microscope attached with a drawing tube. The female epigastric region was dissected and digested in a pancreatin solution (Álvarez-Padilla and Hormiga 2008) for about 3-7 days. The genitalia was mounted on a slide and illustrated as described above. Digital images of the specimens were taken with a Leica MC170 HD camera mounted on a Leica M205C stereomicroscope using the software package Leica Application Suite, LAS version 4.6.2 (Leica Microsystems Limited, Germany). Acquired image stacks of different depths (15 to 50 images per stack) were assembled using Helicon Focus (version 6, Helicon soft Ltd) to create a single image with the entire specimen in focus. All measurements are given in millimeters. All types and voucher specimens are deposited in the National Institute of Fundamental Studies, Kandy, Sri Lanka (NIFS) and the Zoological Research Museum Alexander Koenig, Bonn, Germany (ZFMK).

Abbreviations: Character abbreviations: ALE, Anterior lateral eyes; as, accessory structure; co, conductor; em, embolus; ef, epigastric furrow; gap, globular appendix; lap, lateral apodemes; L, length; ma, embolic accessory appendage; na, nail like process; pof, pore field of receptaculum; pgt, posterior genitalic tube; psg, posterior spiracular groove; psc, paddle-like sclerite; PLE, posterior lateral eyes; PME, posterior median eyes; re, receptaculum; ssa, secretory sac; to, tooth like projection; W, width. Additional abbreviations: FR, Forest reserve.

\section{Taxonomy}

\section{Family Oonopidae Simon, 1890}

\section{Genus Cavisternum Baehr, Harvey \& Smith, 2010}

\section{Cavisternum bom n. sp.}

http://zoobank.org/097D44CE-DFB2-46D8-B688-4188BAFBE2F0 Figs $1 \mathrm{~A}-\mathrm{G}, 2 \mathrm{~A}-\mathrm{B}$

Type material. Male holotype from Sri Lanka, Northern Province, Jaffna District, Mandaitivu FR, 09³6'26"N 79॰59'5"E, 12m, litter; 20-22 September 2016; leg. S. P. Benjamin et al. (IFS_Oon_405) (ZFMK).
Paratype. 1 female; same locality data (IFS_Oon_406) (ZFMK).

Diagnosis. The male of Cavisternum bom n. sp. resembles males of C. attenboroughi Baehr \& Raven, 2013 by the sternal concavity occupying about half the sternal length (Fig. 1D), but differ in having an oval-shaped cymbium-bulb complex with a tube-shaped embolus (Fig. 2A). Females are diagnosed by the epigastric area with narrow copulatory ducts as in C. digweedi Baehr, Harvey $\&$ Smith, 2010, but differ in having a thin semicircular rim, which is widened anteriorly and narrowed posteriorly (see arrow in Fig. 2B).

Etymology. This specific name is a noun in apposition named after "Bom" a magnificent goblin in the story of "The Goblins Looking-Glass" by Enid Blyton (1947).

Description. Male: Total length 1.10 (Carapace, L: 0.40, W: 0.28. Abdomen, L: 0.70, W; 0.32). Coloration: carapace pale orange, sternum pale orange, mouth parts orange-brown, abdominal scuta pale orange, legs yellow, palps pale orange. Carapace ovoid in dorsal view (Fig. 1A), slightly elevated, anteriorly narrowed to half its maximum width, with rounded posterolateral corners, pars cephalica slightly elevated, lateral margin rebordered and surface smooth (Fig. 1C). Clypeus straight in front view, ALE separated from edge of carapace by less than their radius. Six eyes, well developed, ALE largest, circular, PME oval, PLE circular, posterior eye row straight from above, procurved in front view. ALE-ALE separated by less than ALE radius, ALE-PLE separated by less than ALE radius, PME-PME touching, PLE-PME touching. Sternum as longer than wide with concave field of clavate setae occupying about half the sternal length (Fig. 1D). Cheliceral fangs widened. Labium rectangular, not fused to sternum. Endites not excavated distally. Abdomen elongated-oval (Fig. 1B). Dorsal scutum sclerotized, covering full length of abdomen, pale orange, without color pattern, not fused to epigastric scutum. Epigastric scutum sclerotized, ventrally plain, book lung covers large, ovoid, without setae, pedicel tube short, ribbed. Epigastric furrow separates epigastric scutum from postepigastric scutum. Postepigastric scutum long, almost rectangular, covering about more than $3 / 4$ of abdominal length, fused to epigastric scutum, with long posteriorly directed lateral apodemes. Spinneret scutum present as an incomplete ring. Leg spines absent. Genitalia: Palp of normal size, not strongly sclerotized, proximal segments, cymbium, bulb and embolus yellow. Cymbium ovoid in dorsal view, completely fused with bulb without visible seam, bulb oval, bearing a tube-shaped embolus (em) (Fig. 2A).

Female: Total length 1.14 (Carapace, L: 0.44, W: 0.30. Abdomen, L: 0.70, W; 0.32). In general similar to males: Sternum unmodified in females (Fig. 1E); chelicerae smaller. Genitalia: Epigastric area with copulatory duct not reaching posterior spiracular groove, with thin, narrow semicircular rim which is widen anteriorly and narrow posteriorly (Figs 1G, 2B).

Distribution. Known only from the type locality (Fig. 21). 


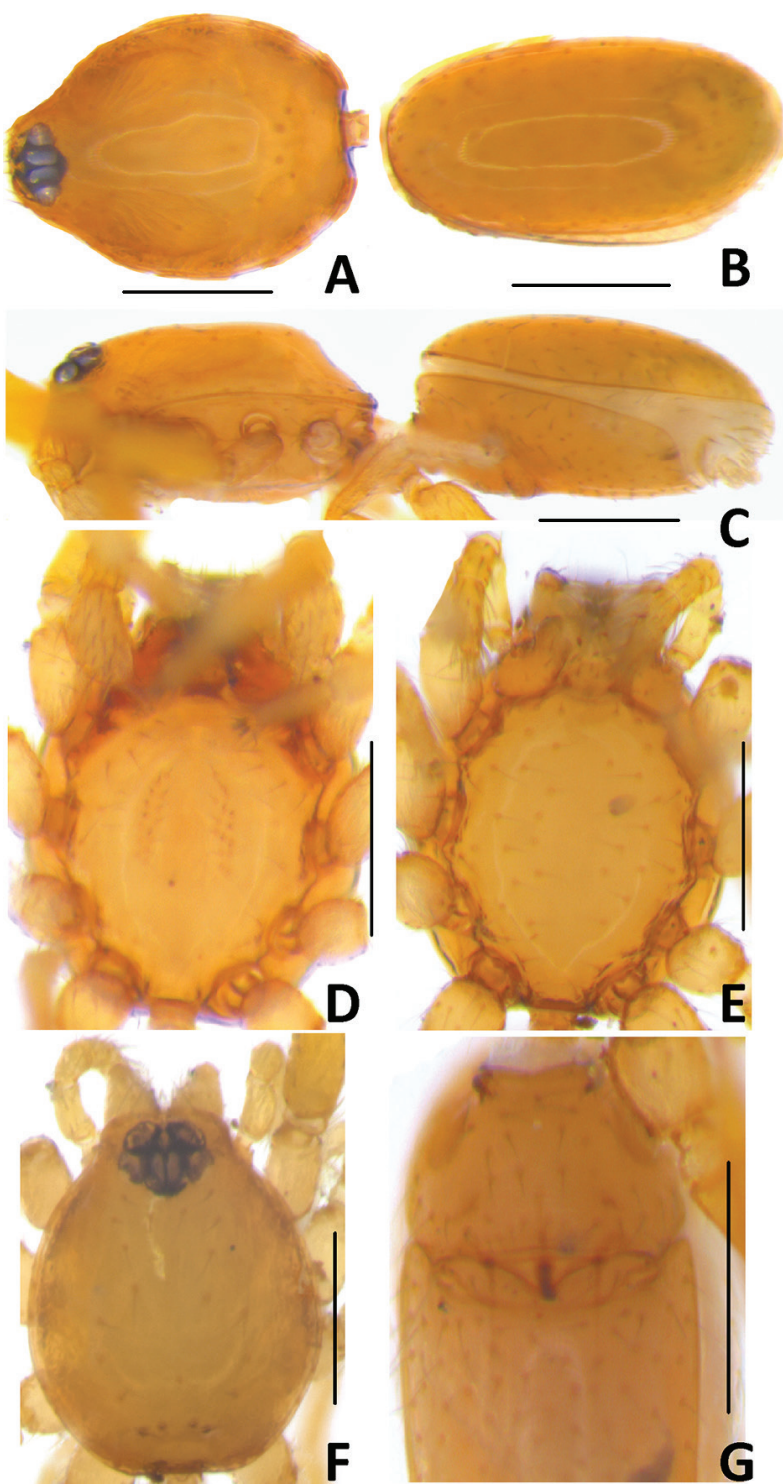

Figure 1. Cavisternum bom n. sp., Male (A-D), female (E-G) from Mandaitivu FR: A, carapace, dorsal view; B, abdomen, dorsal view; C, habitus, lateral view; $\mathbf{D}$, sternum, ventral view; $\mathbf{E}$, same; F, carapace, dorsal view; $\mathbf{G}$, abdomen, ventral view. Scale bars: $0.2 \mathrm{~mm}$.

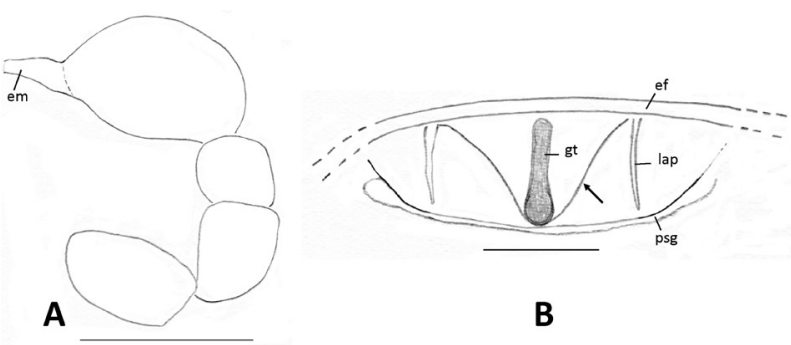

Figure 2. Cavisternum bom n. sp., from Mandaitivue FR: A, male left palp, retrolateral view; B, female epigastric region, dorsal view. Abbreviations: em, embolus; ef, epigastric furrow; lap, lateral apodemes; gt, genitalic tube; psg, posterior spiracular groove. The arrow in (B) points to the thin semicircular rim in the epigastric area. Scale bars: $0.1 \mathrm{~mm}$.

\section{Genus Grymeus Harvey, 1987}

Grymeus dharmapriyai n. sp.

http://zoobank.org/CDD21286-ED78-48F5-B758-63243C16F4F1

Figs $3 \mathrm{~A}-\mathrm{J}, 4 \mathrm{~A}-\mathrm{E}, 5 \mathrm{~A}-\mathrm{B}$

Type material. Male holotype from Sri Lanka, North Western Province, Puttalam District, Wanathavilluwa, $08^{\circ} 10^{\prime} 15^{\prime \prime} \mathrm{N}, 79^{\circ} 52^{\prime} 30^{\prime \prime} \mathrm{E}, 30 \mathrm{~m}$, litter; 24 May 2010; leg. N. Athukorala (IFS_Oon_007) (ZFMK).

Paratypes. 1 male and 3 females; same locality data (IFS_Oon_008-011) (ZFMK).

Other material examined. 2 males; Sri Lanka, Central Province, Kandy District, Gannoruwa FR, $07^{\circ} 16^{\prime} 56.85^{\prime \prime} \mathrm{N}, 80^{\circ} 35^{\prime} 57.25^{\prime \prime} \mathrm{E}, 575 \mathrm{~m}$, litter; 25 September 2013; leg. N. Athukorala et al. (IFS_Oon_156, 166). 1 male; Sri Lanka, Northern Province, Mannar District, surroundings of Vayu resorts-kitesurfing, Thaleimannar, $09^{\circ} 04^{\prime} 56^{\prime \prime} \mathrm{N}, 7^{\circ} 42^{\prime} 4^{\prime \prime} \mathrm{E}, 4 \mathrm{~m}$, litter; 03 April 2018; leg. S.P. Benjamin et al. (IFS_Oon_502).

Diagnosis. The male of $G$. dharmapriyai n. sp. resembles to G. robertsi Harvey, 1987 by carapace without longitudinal rows of long, stout dorsal setae (Fig. 3A) and sperm pore situated in anterior third of abdomen (Fig. $3 \mathrm{H})$, but differ in having the embolic accessory appendage with a dorsal, tooth-like projection (to) and a sharp, pointed embolus (Fig. 5A). Females are recognized by the stout and rounded receptaculum (Fig. 5B).

Etymology. The species is named for the first author's husband P. L. Dharmapriya.

Description. Male: Total length 2.12 (Carapace, L: 0.84, W: 0.34. Abdomen, L: 1.28, W; 0.92). Coloration: uniformly orange brown colored. Carapace pyriform in dorsal view (Fig. 3A), pars cephalica gradually elevated and sudden elevation at middle (Fig. 3C), posterior circular shaped in dorsal view, but anterior rectangular shaped narrowed to less than 0.5 times its maximum width (Fig. $3 \mathrm{~A})$, posterior lateral spikes absent, lateral margin undulated. Clypeus straight in front view, margin unmodified, ALE separated from edge of carapace by about their diameter, setae absent. Six eyes, well developed (Fig. 3D), ALE largest, oval, separated each other by less than their diameter, posterior eyes circular, subequal, touching each other. Sternum heart-shaped (Fig. 3B), sharp margin visible, fused to carapace, radial furrows absent, decorated with rounded spots, lateral margin without indented extensions between coxae, posterior margin not extend. Cheliceral fangs unmodified, labium rounded, anterior invagination of sternum form a pouch (Fig. 3B). Abdomen elongated (Fig. 3G), evenly scattered with short setae, dorsal scutum covering full length of abdomen, no soft tissue visible from above, not fused to epigastric scutum, spinnerets not visible in dorsal view. Epigastric scutum sclertized, book lung covers pale brown, oval shaped, setaceous and not pointed (Fig. 3F). Postepigastric scutum long, fused to epigastric scutum (Fig. 3H). Spinneret scutum present as an incomplete ring. Leg spines absent, short brush like setae on all segments in leg I, II, III and 

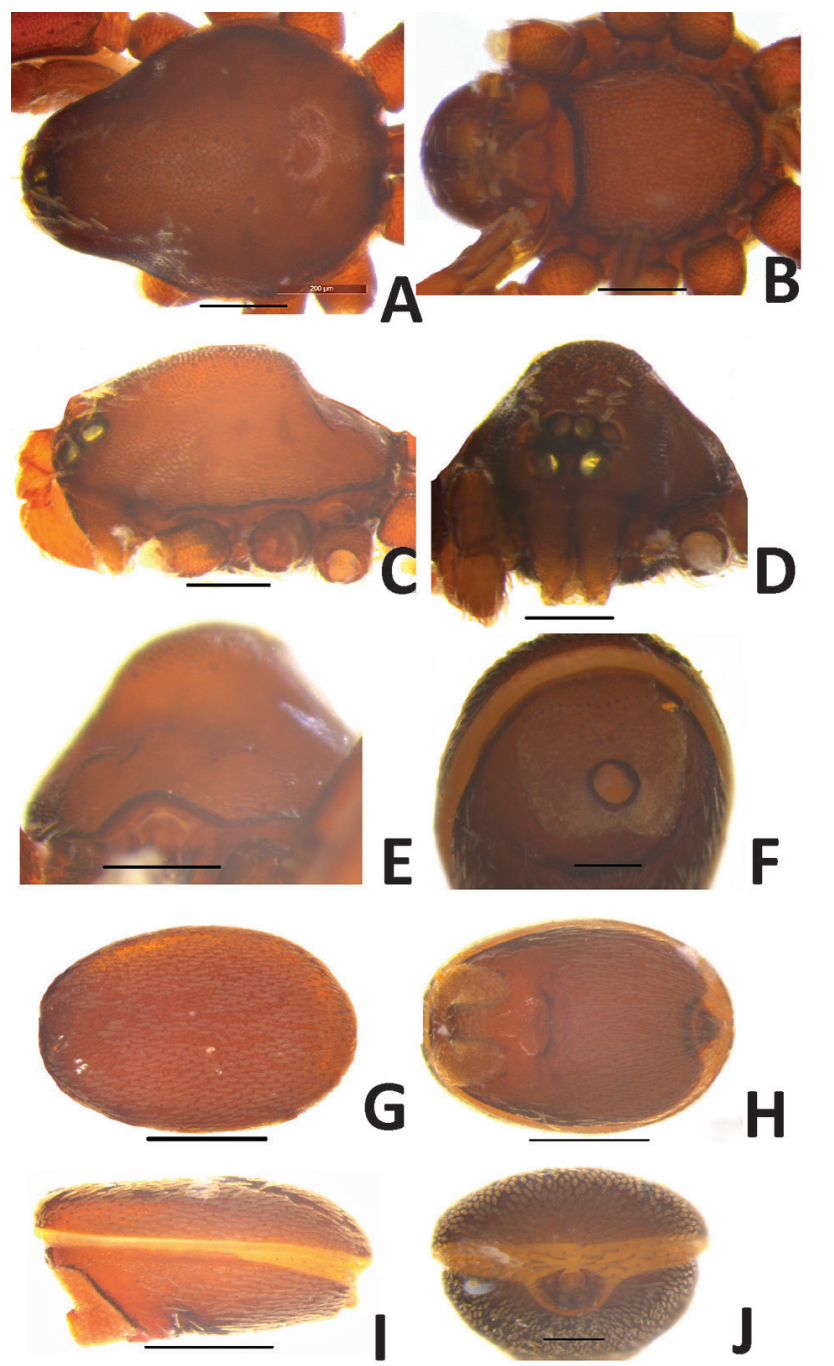

Figure 3. Grymeus dharmapriyai n. sp., male from Wanathavilluwa: A, carapace, dorsal view; $\mathbf{B}$, sternum, ventral view; C, carapace, lateral view; D, same, anterior view; E, same, posterior view; $\mathbf{F}$, abdomen, anterior view; $\mathbf{G}$, same, dorsal view; $\mathbf{H}$, same, ventral view; I, same, lateral view; $\mathbf{J}$, same, posterior view. Scale bars: $0.2 \mathrm{~mm}(\mathbf{A}-\mathbf{F}, \mathbf{J}), 0.5 \mathrm{~mm}(\mathbf{G}-\mathbf{I})$.
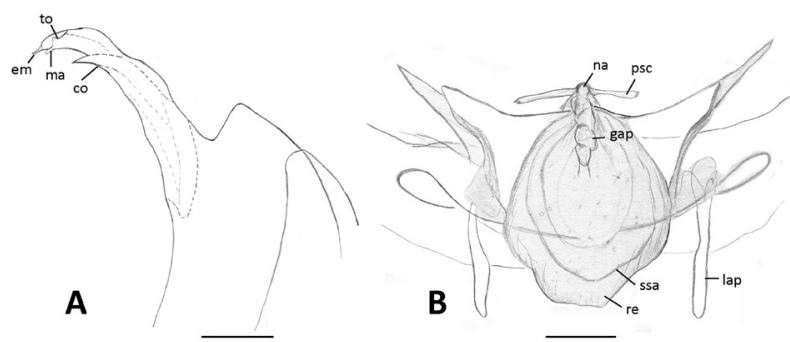

Figure 5. Grymeus dharmapriyai n. sp., from Wanathavilluwa: A, male left palp, distal part, retrolateral view; $\mathbf{B}$, female epigastric region, dorsal view. Abbreviations: co, conductor; em, embolus; gap, globular appendix; lap, lateral apodemes; ma, embolic accessory appendage; na, nail like process; psc, paddle-like sclerite; re, receptaculum; ssa, secretory sac; to, tooth like projection. Scale bars: $0.1 \mathrm{~mm}$.

IV. Genitalia: Palp of normal size, not strongly sclerotized, cymbium not extending beyond bulb, covered $2 / 3$ of the bulb. Embolus (em), embolic accessory appendage (ma) and conductor (co) sharp and pointed, distal part of embolic accessory appendage bears a tooth-like projection (to) dorsally, slightly shorter than embolus, conductor short, curved ventrally (Fig. 5A).

Female: Total length 3.10 (Carapace, L:1.22, W:0.82; Abdomen, L: 1.80, W: 1.24). In general similar to males, larger than male, pars cephalica elevated from in lateral view, surface sides granulated (Figs 4A, 4C), lateral margin slightly undulated, sternum with hairs, not decorated with rounded spots (Fig. 4D), setae presence on chelicera, book lung covers small, narrowed, differ from male (Fig. 4B). Genitalia: Receptaculum (re) stout, rounded shaped, secretory sac (ssa) oval, pore field of receptaculum (pof) concentrated at middle of secretory sac, globular appendix (gap) short, less than 0.5 times the length of receptaculum, with an anterior paddle like sclerite (psc) and a nail like process (na), with lateral sclerites, with lateral apodemes (lap) (Fig. 5B).

Distribution. Wanathavilluwa, Gannoruwa FR, Thaleimannar (Fig. 21).

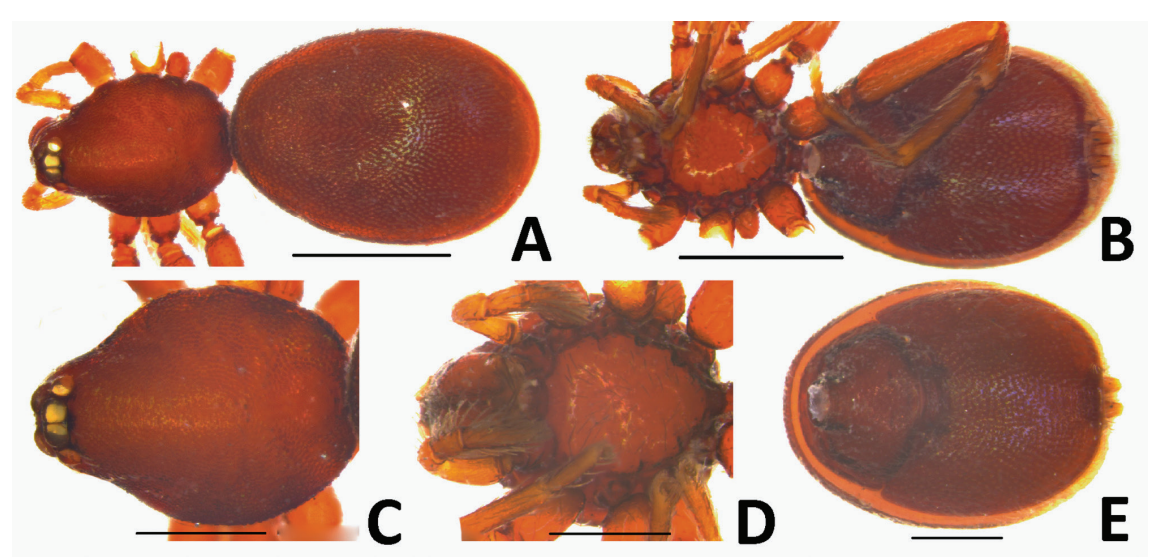

Figure 4. Grymeus dharmapriyai n. sp., female from Wanathavilluwa: A, habitus, dorsal view; B, same, ventral view; C, carapace, dorsal view; D, sternum, ventral view; E, abdomen, ventral view. Scale bars: $1 \mathrm{~mm}(\mathbf{A}-\mathbf{B}), 0.5 \mathrm{~mm}(\mathbf{C}-\mathbf{E})$. 


\section{Genus Ischnothyreus Simon, 1893}

\section{Ischnothyreus chippy n. sp.}

http://zoobank.org/BF3D371F-2E39-405A-84CB-1A8F5D41338B Figs $6 \mathrm{~A}-\mathrm{C}, 7 \mathrm{~A}-\mathrm{B}$

Type material. Male holotype from Sri Lanka, Uva Province, Badulla District, $189^{\text {th }}$ mile post, Between Koslanda and Beragala, $06^{\circ} 44^{\prime} 48.4^{\prime \prime} \mathrm{N} 80^{\circ} 57^{\prime} 56.3^{\prime \prime} \mathrm{E}, 1370 \mathrm{~m}, 01$ January 2012, leg. S. P. Benjamin et al., General Collecting. (IFS_Oon 132) (ZFMK).

Diagnosis. Males are easily identified by medially constricted abdomen in lateral view (Fig. 6C).

Etymology. This species name is a noun in apposition named after "Chippy" a brownie and one of the characters in the story "Billy's Little Boats" by Blyton (1971). Brownies possess queer little pointed feet like goblins.

Description. Male: Total length 1.66 (Carapace, L: 0.74, W: 0.52. Abdomen, L: 0.92, W; 0.32).

Coloration: carapace brown, sternum and mouthparts orange-brown, abdominal scuta brown, legs yellow-brown, palps pale dark brown. Carapace ovoid (Fig. $6 \mathrm{~A})$, with dark brown egg-shaped patches behind eyes, anteriorly narrowed to half its maximum width with rounded posterolateral corners, pars cephalica slightly elevated (Fig. 6A). Clypeus straight in front view, ALE separated from edge of carapace by less than their radius. Six eyes, well developed, ALE largest, circular, PME and PLE circular, posterior eye row procurved in front view. ALE-ALE separated by less than ALE radius, ALE-PLE

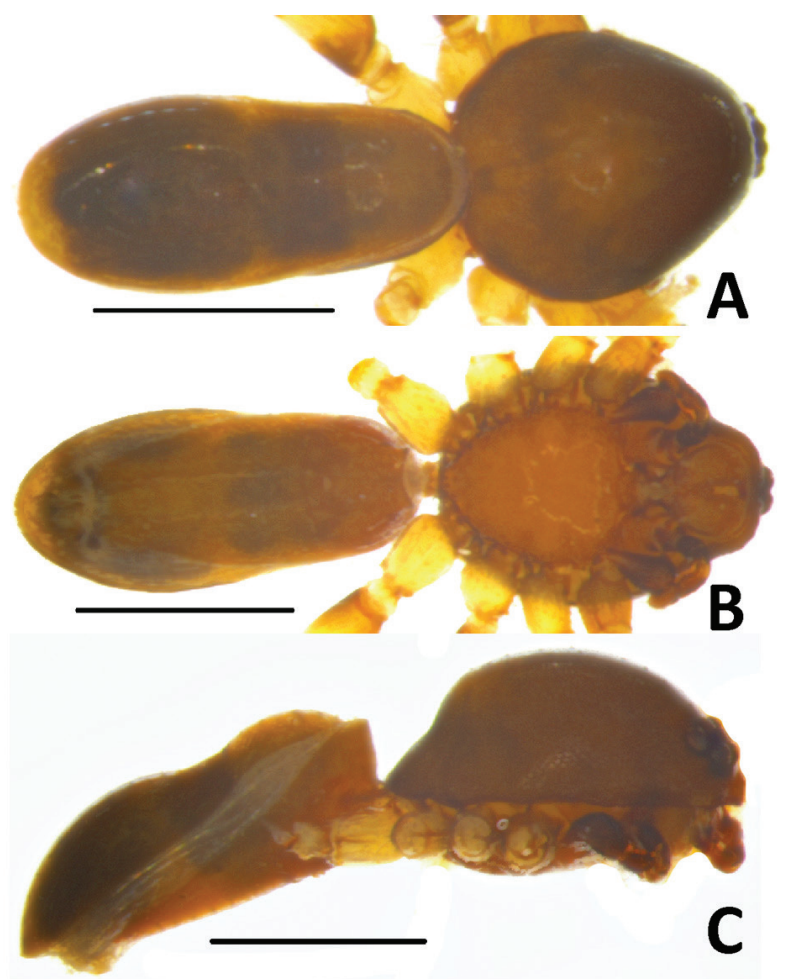

Figure 6. Ischnothyreus chippy n. sp., male from a forest between Koslanda and Beragala: A, habitus, dorsal view; B, same, ventral view; $\mathbf{C}$, same, lateral view. Scale bars: $0.5 \mathrm{~mm}$.
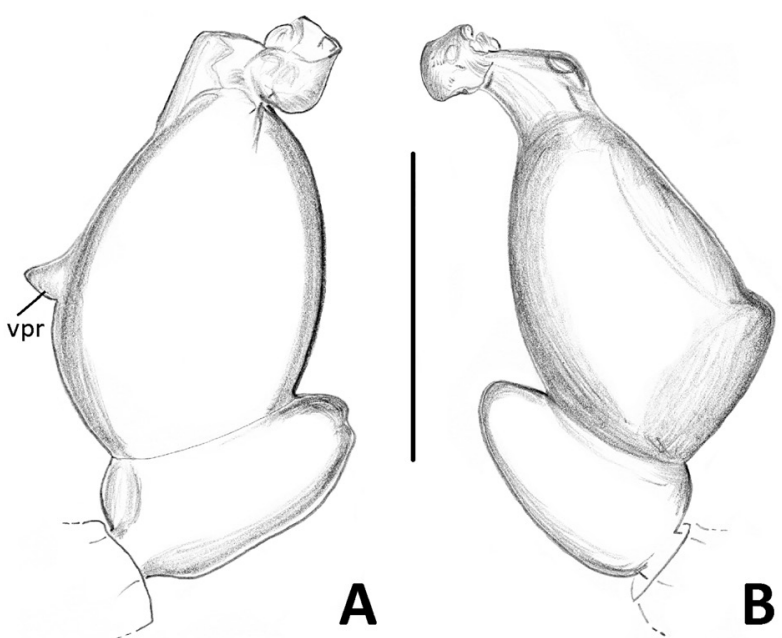

Figure 7. Ischnothyreus chippy n. sp., male from a forest between Koslanda and Beragala: A, left palp, retrolateral view; B, same, prolateral view. Abbreviation: vpr, ventral protuberance. Scale bars: $0.1 \mathrm{~mm}$.

separated by less than ALE radius, PME-PME touching, PLE-PME touching. Sternum as longer than wide (Fig. 6B). Cheliceral fangs widened. Endites not excavated distally. Abdomen elongated-oval (Fig. 6A), constricted medially in lateral view (Fig. 6C), dorsal scutum sclerotized, covering full length of abdomen with brown and dark-brown color pattern, not fused to epigastric scutum (Fig. 6A). Epigastric scutum sclerotized, ventrally plain, book lung covers large, elliptical, without setae, pedicel tube short, ribbed. Epigastric furrow separates epigastric scutum from postepigastric scutum. Postepigastric scutum long, covering all abdominal length, fused to epigastric scutum. Leg spines present, four spines present on tibia III (Leg I, II missing). Genitalia: Palp of small size, strongly sclerotized. Cymbium ovoid, completely fused with bulb without visible seam, bulb oval, with ventral protuberance (vpr) and tip with multiple, complex processes (Figs 7A, B).

Female: Unknown.

Distribution. Known only from the type locality (Fig. 21).

\section{Genus Opopaea Simon 1892}

\section{Opopaea spinosiscorona $\mathbf{n}$. sp.}

http://zoobank.org/E5E2F6B7-8511-46E0-8C26-F88B0162716A

Figs $8 \mathrm{~A}-\mathrm{J}, 9 \mathrm{~A}-\mathrm{B}, 10 \mathrm{~A}-\mathrm{B}$

Type material. Male holotype from Sri Lanka, Sabaragamuwa Province, Kegalle District, Kurulukelle FR, $07^{\circ} 14^{\prime} 12^{\prime \prime} \mathrm{N}, 80^{\circ} 20^{\prime} 33^{\prime \prime} \mathrm{E}, 200 \mathrm{~m}$, litter; 12 November 2014; leg. N. Athukorala (sample IFS Oon 252) (ZFMK).

Paratypes. 4 males, 1 female; same locality data (IFS_Oon_247-251) (ZFMK).

Other material examined. 1 female; Sri Lanka, Central Province, Kandy District, Meemure, 07² $14^{\prime} 14^{\prime \prime} \mathrm{N}$ 80³8'19"E, 890m, 20 April 2010, leg. S. Batuwita, Gu- 


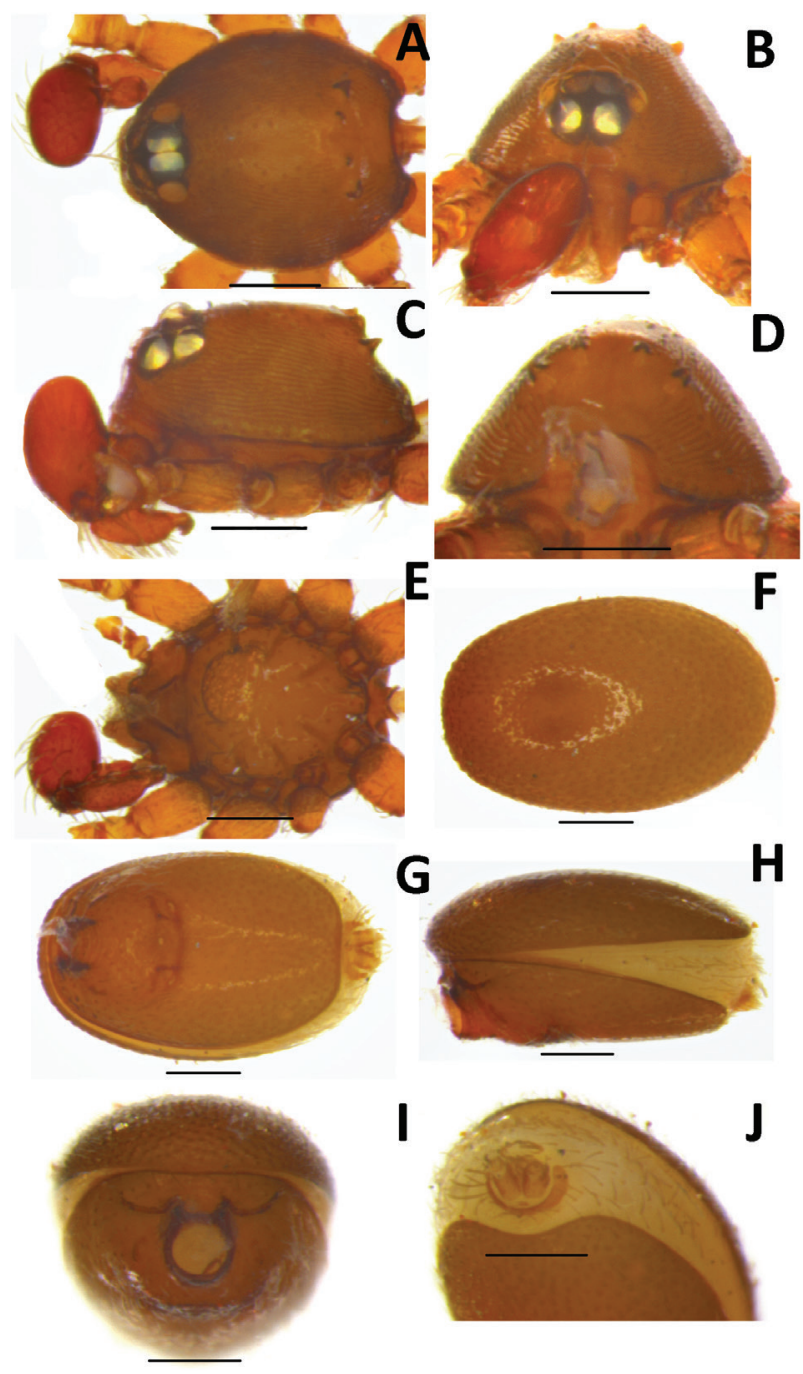

Figure 8. Opopaea spinosiscorona n. sp., male from Kurulukelle FR: A, carapace, dorsal view; B, same, anterior view; C, same, lateral view; D, same posterior view; E, sternum, ventral view; F, abdomen, dorsal view; $\mathbf{G}$, same, ventral view; H, same, lateral view; I, same, anterior view; J, same, posterior view. Scale bars: $0.2 \mathrm{~mm}$.

nawardana (IFS_Oon_023). 1 male, 1 female; Deenston, Knuckles range, $07^{\circ} 20^{\prime} 10^{\prime \prime} \mathrm{N}, 80^{\circ} 51^{\prime} 31^{\prime \prime} \mathrm{E}, 1120 \mathrm{~m}, 19$ January 2010, leg. S. P. Benjamin, S. Batuwita (IFS Oon_061-062). 4 males, 3 females; Udawattakele FR, $07^{\circ} 17^{\prime} 57^{\prime \prime} \mathrm{N}, 80^{\circ} 38^{\prime} 29^{\prime \prime} \mathrm{E}, 580 \mathrm{~m}, 21$ August 2012, leg. S. P. Benjamin, 24 August 2016, N. Athukorala et al. (IFS Oon_109-114, 377-378). 1 male, 2 females; Gannoruwa FR, $07^{\circ} 16^{\prime} 56.85^{\prime \prime} \mathrm{N}, 80^{\circ} 35^{\prime} 57.25^{\prime \prime} \mathrm{E}, 575 \mathrm{~m}, 25$ November 2013, leg. N. Athukorala et al. (IFS_Oon_157-159). 1 female, 1 male; Dunumadalawa $\overline{F R}, 0 \overline{7}^{\circ} 17^{\prime} 00^{\prime \prime} \mathrm{N}$, $80^{\circ} 37^{\prime} 49^{\prime \prime}$ E, 600m, 26 November 2013, leg. S. P. Benjamin et al.; 05 January 2017, N. Athukora et al. (IFS Oon_187, 431). 2 males; Knuckles site 01 (Riverston), 19 February 2015, leg. N. Athukorala (IFS_Oon_282283). 3 males, 1 female; Matale District, IFS Arboretum, 0751'34"N, 8040'28"E, 180m, 24 August 2010, leg. S. P. Benjamin,S. Batuwita; 07 July 2013, leg. S. P. Benja- min, N. Athukorala; 27 April 2010, leg. S. P. Benjamin, S. Batuwita; 20 January 2016, leg. N. Athukorala et al. (IFS_Oon_048, 069, 073, 339). 1 male; Riverston, Knuckles range, $07^{\circ} 31^{\prime} 42^{\prime \prime} \mathrm{N}, 80^{\circ} 44^{\prime} 17^{\prime \prime} \mathrm{E}, 1100 \mathrm{~m}, 02$ December 2009, leg. S. P. Benjamin, S. Batuwita (IFS_Oon_049). 2 males; Elahara/Pallegama Road, $3 \mathrm{~km}$ to Pallegama, $07^{\circ} 32^{\prime} 16^{\prime \prime} \mathrm{N}, 80^{\circ} 40^{\prime} 20^{\prime \prime} \mathrm{E}, 267 \mathrm{~m}, 03$ February 2016, leg. S. P. Benjamin, N. Athukorala (IFS_Oon_318-319). 1 male, 1 female; Bowatenna Reservoir area, Thick forest along road, $07^{\circ} 39^{\prime} 37^{\prime \prime} \mathrm{N}, 80^{\circ} 41^{\prime} 18^{\prime \prime} \mathrm{E}, 252 \mathrm{~m}, 10$ February 2016, leg. S. P. Benjamin et al. (IFS_Oon_331-332). 3 males, 2 females; Uva Province, Badulla District, forest around Rawana Cave, 06 $51^{\prime} 52.4^{\prime \prime} \mathrm{N} 81^{\circ} 03^{\prime} 01.3^{\prime \prime} \mathrm{E}, 990 \mathrm{~m}, 31$ December 2011, leg. S. P. Benjamin et al. (IFS_Oon_218222). 1 female; Monaragala District, Westminister Abbey, $07^{\circ} 02^{\prime} 42^{\prime \prime} \mathrm{N}, 81^{\circ} 032^{\prime} 16^{\prime \prime} \mathrm{E}, 120 \mathrm{~m}, 10$ February 2010, leg. S. P. Benjamin, S. Batuwita (IFS_Oon_077). 1 female; North Central Province, Anuradahapura District, Mihinthale FR, $08^{\circ} 21^{\prime} 10.60^{\prime \prime} \mathrm{N}, 80^{\circ} 30^{\prime} 14.54^{\prime \prime} \mathrm{E}, 06$ July 2014, leg. N. Athukorala et al. (IFS_Oon_229). 1 female; Sabaragamuwa Province, Kegalle District, Kurulukele FR, $07^{\circ} 14^{\prime} 12^{\prime \prime} \mathrm{N}, 80^{\circ} 20^{\prime} 33^{\prime \prime} \mathrm{E}, 200 \mathrm{~m}, 21$ August 2010, leg. S. Batuwita (IFS_Oon_030). 1 male; Eastern Province, Ampara District, $\bar{N}$ uwaragala FR, $07^{\circ} 26^{\prime} 07.4^{\prime \prime} \mathrm{N}, 81^{\circ} 31^{\prime} 58.8^{\prime \prime} \mathrm{E}$, 70m, 19 May 2015, leg. N. Athukorala (IFS_Oon_295). 2 females North Western Province, Kurunagala District, Ethagala FR, $07^{\circ} 28^{\prime} 17^{\prime \prime} \mathrm{N}, 80^{\circ} 22^{\prime} 30^{\prime \prime} \mathrm{E}, 190 \mathrm{~m}, 20$ August 2010, leg. S. Batuwita (IFS_Oon_058-059).

Diagnosis. Both males and females of Opopaea spinosiscorona $\mathrm{n}$. sp. are easily identified from all other species of the genus by the presence of four triangular-shaped spines on the posterior part of the carapace (Fig. 8A). Further, males of $O$. spinosiscorona $\mathrm{n}$. $\mathrm{sp}$. can be separated from other species of the genus by the enlarged palpal tip and short and beak-like extensions of the tip (Fig. 10A). Females can be identified by the U-shaped ridge between the epigastric furrow and the posterior spiracular groove which is shorter than the lateral apodemes (Fig. 10B).

Etymology. The species name refers to the presence of four spines on its carapace.

Description. Male: Total length 1.62 (Carapace, L: 0.64, W: 0.56. Abdomen, L: 0.98, W; 0.64). Coloration: uniformly orange brown colored, palps reddish brown. Carapace ovoid in dorsal view (Fig. 8A), pars cephalica slightly elevated in lateral view (Fig. 8C), with angular posterolateral corners, posterolateral edge without pits, anterolateral corners without extension or projections, posterolateral surface with four triangular spikes (Fig. 8A), sides striated (Fig. 8C), lateral margin straight. Clypeus straight in front view, margin unmodified, ALE separated from edge of carapace by more than their diameter, median projection absent, setae present (Fig. 8B). Six eyes, well developed (Fig. 8B), ALE largest, PLE smallest, ALE round, PME oval, posterior eye row curved from above, procurved from front; all eyes touching each other. Sternum long as wide, uniform, fused to carapace, median concavity absent, with radial furrows between coxae I-II, II-III, III-IV (Fig. 8E), furrow wrinkled, sur- 


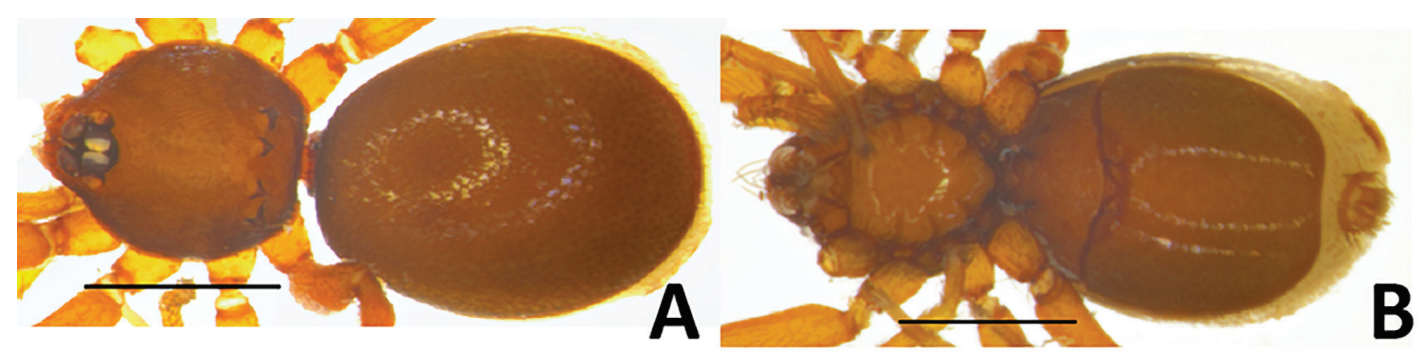

Figure 9. Opopaea spinosiscorona n. sp., female from Kurulukelle FR: A, habitus, dorsal view; B, same, ventral view. Scale bars: $0.5 \mathrm{~mm}$.

face smooth, with pits, posterior margin not extending posteriorly of coxae IV (Fig. 8E).

Chelicerae fangs straight, anterior face unmodified, without tooth-like projections, chelicerae inner margin with enlarged setae (ceh). Labium triangular, fused to sternum, anterior margin indented at middle. Endites not excavated distally. Abdomen elongated oval, rounded posteriorly (Fig. 8F). Dorsal scutum strongly sclerotized, orange-brown, without color pattern, covering full length of abdomen, no soft tissue visible from above (Fig. 8 F). Epigastric scutum strongly sclerotized, surrounding pedicel, not protruding (Fig. 8G) book lung covers small, straight, without setae posterior spiracles connected by groove (Fig. 8G). Postepigastric scutum long, semicircular, covering nearly full length of abdominal length, fused to epigastric scutum, anterior margin unmodified, with short posteriorly directed lateral apodemes (Fig. 8G). Pedicel tube short, with small, dorsolateral, triangular extensions, scuto-pedicel region with paired curved scutal ridges (Fig. 8I). Spinneret scutum present as an incomplete ring (Fig. 8J), with fringe of long setae. Leg spines absent, femur IV not thickened, but longer than femora I-III. Genitalia: Sperm pore small, oval, situated at level of anterior spiracles (Fig. 8G). Palp slightly sclerotized (Fig. 8A), proximal segments pale orange; femur two or more times as long as trochanter, attaching to patellae subbasally, patellae longer and larger than femur, cymbium pale orange, narrow in dorsal view, completely fused with bulb, no seam visible, not extending beyond distal tip of bulb, bulb pale orange, 1 to 1.5 times as long as cymbium, slender, elongated, ventrally expanded (Fig. 10A).

Female: Total length 1.74 (Carapace, L: 0.64, W: 0.56. Abdomen, L: 1.10, W; 0.72). In general similar to males. Carapace anterior narrowed to 0.49 times its maximum width (Fig. 9A). Dorsal scutum covering most of abdomen, postepigastric scutum covering most of abdomen, not fused to epigastric scutum. Genitalia: Epigastric region with V-shaped ridge between epigastric furrow and Posterior spiracular groove in ventral view (Fig 9B).

Distribution. Kurulukelle FR, Udawattakele FR, Gannoruwa FR, Ethagala FR, Nuwaragala FR, Mihinthale FR, Westminister Abbey, forest around Rawanaella Cave, Bowatenna Reservoir area, Dambulla IFS Arboretum, Knuckles range (Deenston, Meemure, Riverston), Dunumadalawa FR.

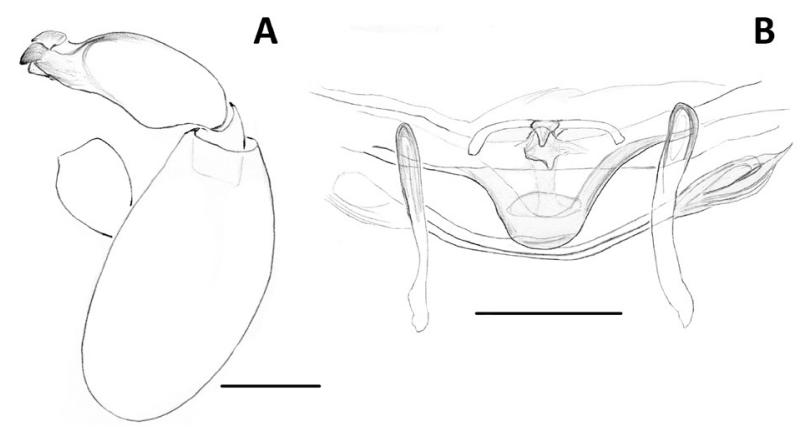

Figure 10. Opopaea spinosiscorona n. sp., from Kurulukelle FR: A, male left palp, prolateral view; $\mathbf{B}$, female epigastric region, ventral view. Scale bars: $0.1 \mathrm{~mm}$.

\section{Genus Pelicinus Simon, 1892}

\section{Pelicinus snooky n. sp.}

http://zoobank.org/36E464E3-3DF0-4ABA-8305-1FF3CFFEA95D (Figs 11A-J, 12A-D, 13A-C)

Type material. Male holotype from Sri Lanka, North Western Province, Kurunegala District, Ethagala FR, 07²9'11.23"N 80²2'21.64"E, 190 m, litter; 08 April 2015; leg. S. P. Benjamin et al. (IFS_Oon_272) (ZFMK).

Paratype. 1 female; same locality data (IFS_Oon_050) (ZFMK).

Diagnosis. Pelicinus snooky n. sp. resembles to $P$. schwendingeri Platnick et al., 2012, by presence of transverse ridges on scuto-pedicel region (Fig. 11F), but can be differentiated by dorsally directed, sclerotized blunt hornshaped embolus with a squared accessory structure (Fig. 13A). Females can be identified by anteriorly directed narrow, cylindrical tube near to epigastric furrow (Fig. 13C).

Etymology. This species a noun in apposition named after "Snooky" a goblin and a main character in the story "The Firework Goblins" written by Blyton in 1971.

Description. Male: Total length 1.16. (Carapace, L: 0.48, W: 0.44. Abdomen, L: 0.68, W: 0.41). Coloration: carapace orange-brown, sternum and mouthparts orange-brown, abdominal scuta orange-brown, legs orange, palps pale orange. Carapace pyriform in dorsal view (Fig. 11A), undulated posteriorly, narrowed anteriorly (Fig. 11A), slightly elevated in lateral view 


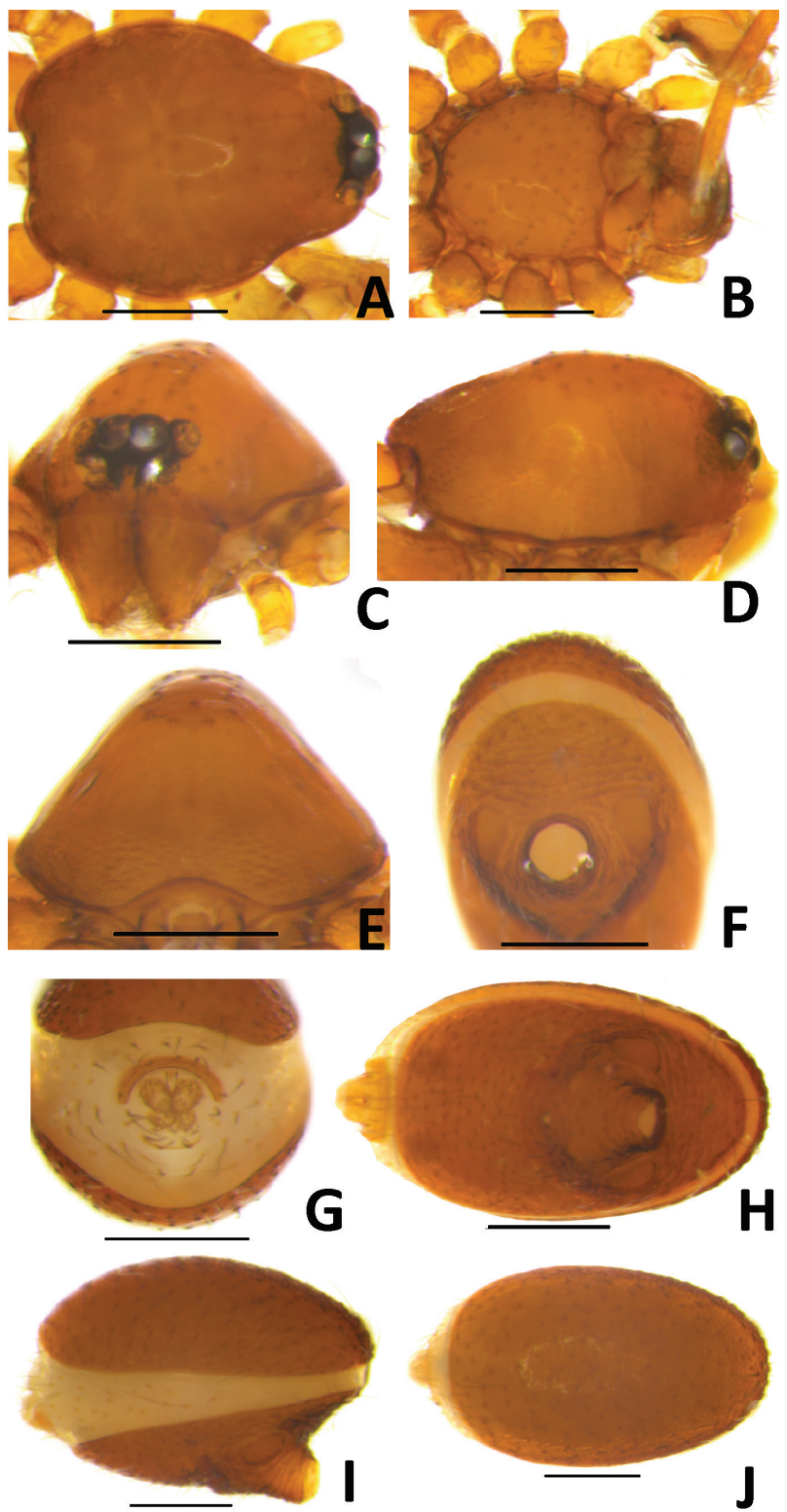

Figure 11. Pelicinus snooky n. sp., male from Ethagala FR: A, carapace, dorsal view; B, sternum, ventral view; $\mathbf{C}$, carapace, anterior view; D, same, lateral view; E, same, posterior view; F, abdomen, anterior view; G, same, posterior view; H, same, ventral view; I, same, lateral view; J, same, dorsal view. Scale bars: $0.2 \mathrm{~mm}$.

(Fig. 11D), surface and sides smooth, slightly granulated posteriorly, lateral margin without denticles (Fig. 11D). Clypeus straight in front view, widen, slightly rebordered. Chelicerae straight, anterior face unmodified (Fig. 11C). Six eyes, well developed (Fig. 11C), PME largest, all oval, ALE separated by more than their diameter, ALE-PLE separated by less than ALE radius, PME touching throughout most of their length. Sternum as long as wide, radial furrows in between coxae absent, surface smooth, pits are evenly scattered (Fig. 11B). Abdomen ovoid, rounded posteriorly, small rounded pits visible through dorsal scutum (Fig. 11J), cover full

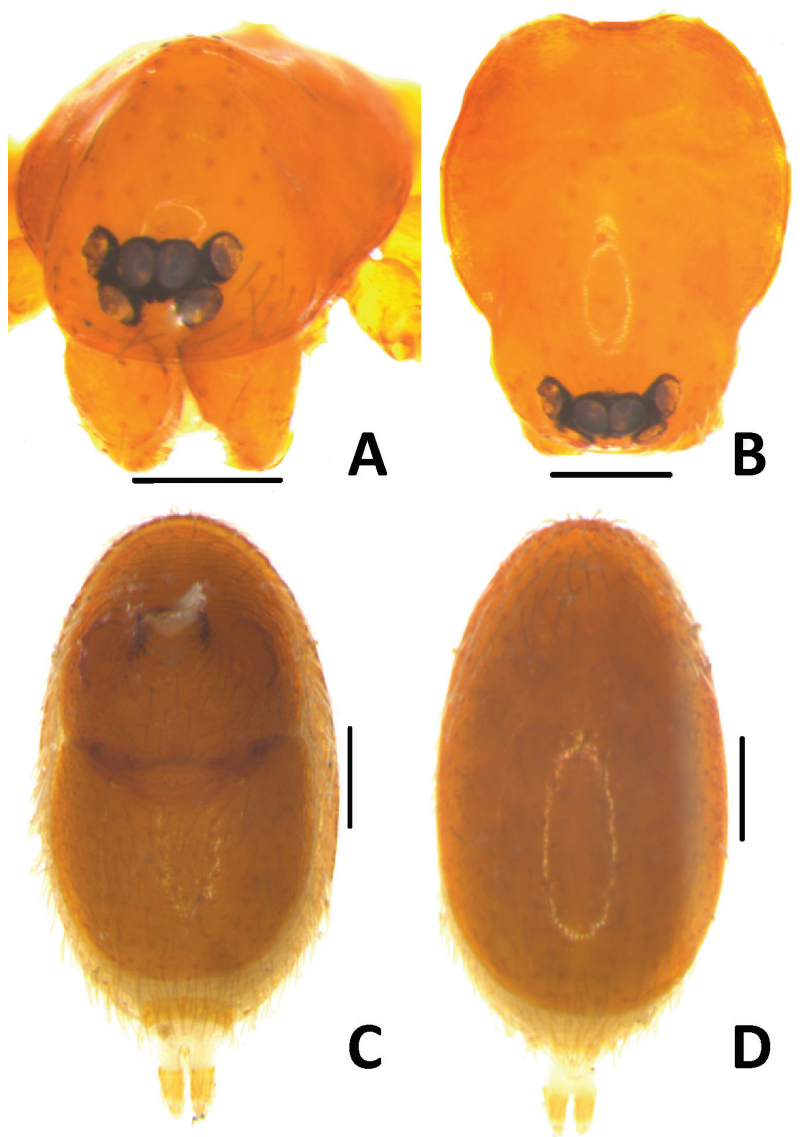

Figure 12. Pelicinus snooky n. sp., female from Ethagala FR: A, carapace, anterior view; B, same, dorsal view; C, abdomen, ventral view; D, same, dorsal view. Scale bars: $0.2 \mathrm{~mm}$ (A-D).

length of abdomen, soft tissue visible from above posteriorly. Postepigastric scutum long, rectangular, fused to epigastric scutum, without posteriorly directed lateral apodemes (Fig. 11H). Posterior spiracles connected by groove (Fig. 11H) booklung covers large, ear-shaped, pedicel tube short, scuto-pedicel region with many strongly wrinkled ridges (Fig. 11F). Spinnerets scutum incomplete (Fig. 11G). Leg spines absent. Genitalia: Sperm pore large, situated at level of anterior spiracular groove (Fig. 11H). Palp of normal size, not strongly sclerotized, femur two or more times as long as trochanter, patellae shorter than femur, tibia with three trichobothria. Cymbium yellow, narrow in dorsal view, not fused with bulb. Bulb stout, pea-shaped, 1.5 times than cymbium, with a dorsally directed, sclerotized blunt horn-shaped embolus (em) with a squared accessory structure (as) (Fig. 13A).

Female: Total length 1.86 (Carapace, L: 0.72, W: 0.58. Abdomen, L: 1.14, W; 0.68). In general similar to males (Figs. 12A-D). Genitalia: Anteriorly directed narrow, cylindrical genitalic tube (gt) near to epigastric furrow (ef) with broad, boat shaped receptaculam (re) (Fig. 13C).

Distribution. Known only from the type locality (Fig. 21). 


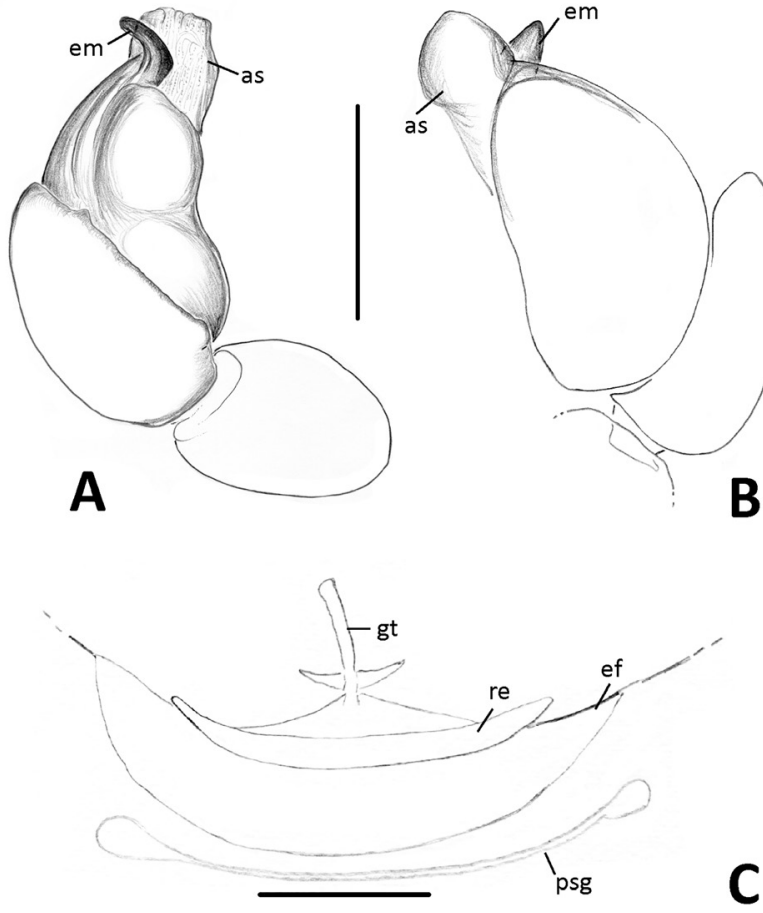

Figure 13. Pelicinus snooky n. sp., from Ethagala FR: A, left palp, prolateral view; B, male left palp, retrolateral view; $\mathbf{C}$, female epigastric region, dorsal view. Abbreviations: as, accessory structure; em, embolus; ef, epigastric furrow; gt, genitalic tube; psg, posterior spiracular groove; re, receptaculum. Scale bars: $0.1 \mathrm{~mm}$.

\section{Pelicinus tumpy n. sp.}

http://zoobank.org/DF94A033-C0CD-49D5-9AB8-9B67A6B96E70 Figs $14 \mathrm{~A}-\mathrm{H}, 15 \mathrm{~A}-\mathrm{D}$

Type material. Male holotype from Sri Lanka, North Central Province, Anuradhapura District, Mihinthale FR, 08 $21^{\prime} 10.60^{\prime \prime} \mathrm{N} 80^{\circ} 30^{\prime} 14.54^{\prime \prime} \mathrm{E}, 123 \mathrm{~m}$, litter; 06 July 2014; leg. N. Athukorala, C. I. Clayton (IFS_Oon_263) (ZFMK).

Paratype. 1 female; same locality data; (IFS Oon_264). 1 male; same locality data, 14 June 2016, leg. N. Athukorala et al. (IFS_Oon_372) (ZFMK).

Diagnosis. Males of Pelicinus tumpy n. sp. can be identified by the prolaterally curved embolus, with distally narrowed, bifid accessory structure and a conductor (Fig. 15B). Females can be identified by the short, convoluted posterior genitalic tube in between posterior spiracular groove and epigastric furrow (Fig. 15C).

Etymology. This species is a noun in apposition named after "Tumpy" a little goblin in the story, The Goblins Looking-Glass by Blyton (1947).

Description. Male: Total length 1.32 (Carapace, L: 0.60, W: 0.48. Abdomen, L: 0.72, W: 0.46). Coloration: carapace orange-yellow, sternum and mouthparts orange, abdominal scuta orange-yellow, abdominal inter scutal region white and covered with setae, legs pale orange, palps pale orange. Carapace pyriform in dorsal view, straight
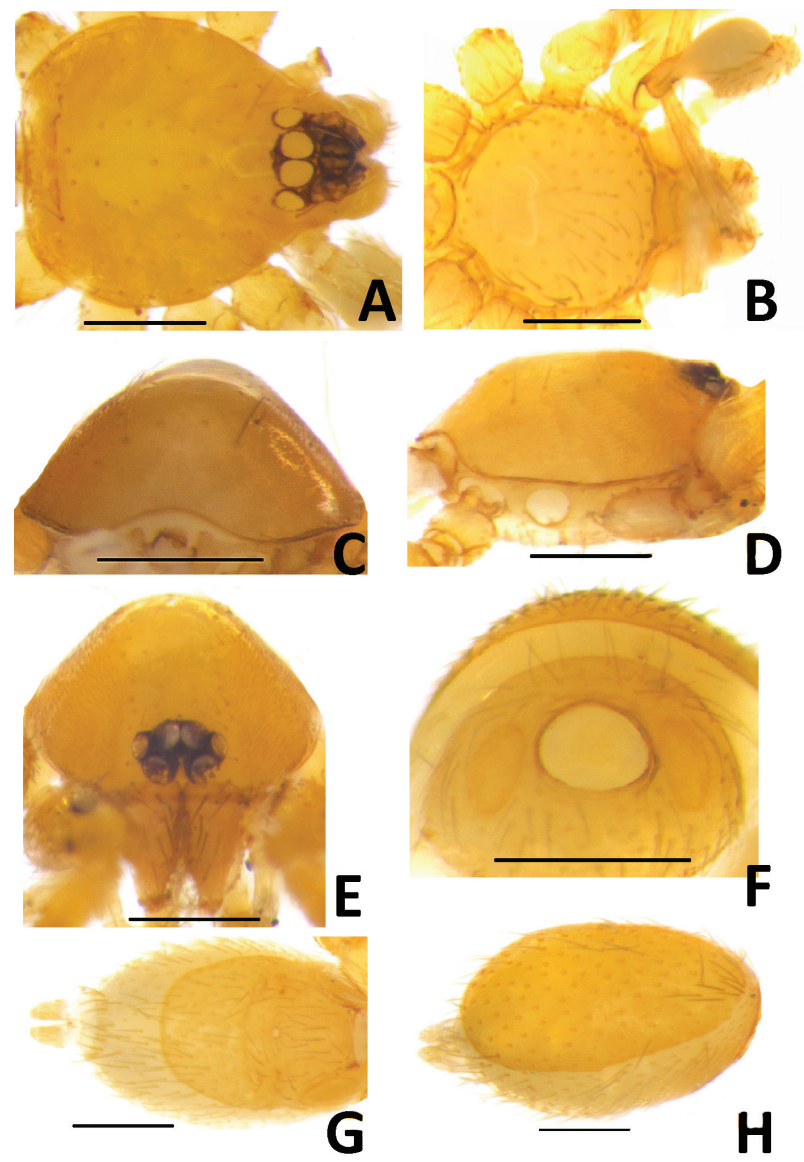

Figure 14. Pelicinus tumpy n. sp., male from Mihinthale FR: A, carapace, dorsal view; B, sternum, ventral view; $\mathbf{C}$, carapace, posterior view; D, same, lateral view; E, same, anterior view; F, abdomen, anterior view; G, same, ventral view; H, same, dorsal view. Scale bars: $0.2 \mathrm{~mm}$.

posteriorly, narrowed anteriorly (Fig. 14A), slightly elevated in lateral view (Fig. 14D), surface and sides finely lined, lateral margin without denticles (Fig. 14D). Clypeus straight in front view, slightly rebordered. Six eyes, well developed (Fig. 14E), PME largest, all oval, ALE separated by more than their diameter, ALE-PLE separated by less than ALE radius, PME touching throughout most of their length. Sternum as long as wide, radial furrows in between coxae absent, surface smooth, pits are evenly scattered (Fig. 14B). Chelicerae fangs straight, anterior face unmodified (Fig. 14E). Abdomen ovoid, with small rounded pits visible through dorsal scutum (Fig. 14H), cover full length of abdomen, no soft tissue visible from above. Postepigastric scutum long, rectangular, fused to epigastric scutum, without posteriorly directed lateral apodemes (Fig. 14G). Posterior spiracles connected by groove (Fig. 14G). booklung covers large, ovoid, pedicel tube short, unmodified, scuto-pedicel region unmodified (Fig. 14F). Spinnerets scutum absent. Legs spines absent. Genitalia: Sperm pore large, situated at level of anterior spiracular groove (Fig. 14G). Palp of normal size, not strongly sclerotized femur two or more times as long as trochanter, patellae shorter than femur, tibia with three trichobothria. Cym- 

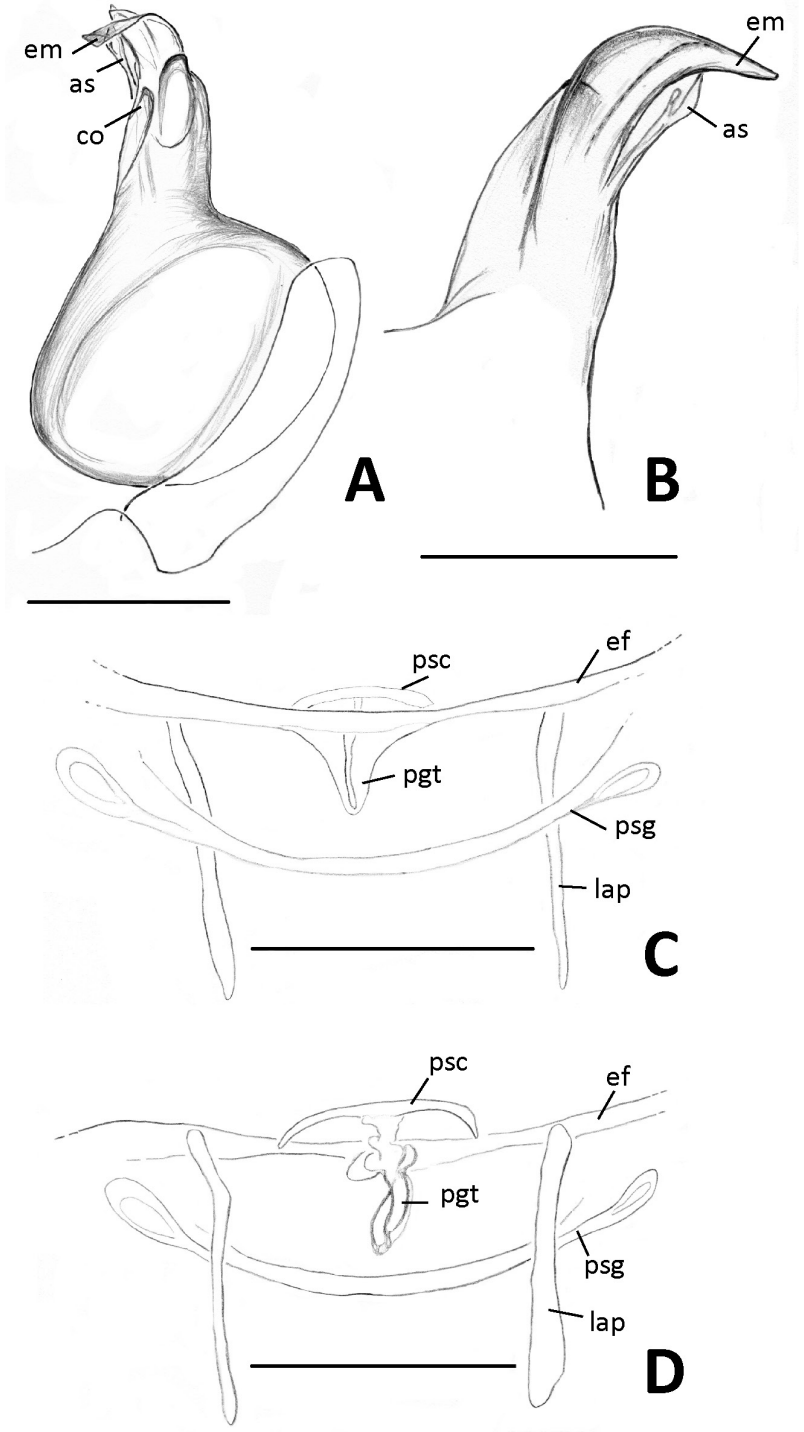

Figure 15. Pelicinus tumpy n. sp., from Mihinthale FR: A, male left palp, retrolateral view; $\mathbf{B}$, details of the distal part of the bulb; $\mathbf{C}$, female epigastric region, dorsal view; $\mathbf{D}$, female epigastric region, ventral view. Abbreviations: as, accessory structure; co, conductor; em, embolus; ef, epigastric furrow; lap, lateral apodemes; pgt, posterior genitalic tube; psg, posterior spiracular groove; psc, paddle-like sclerite. Scale bars: $0.1 \mathrm{~mm}$.

bium yellow, narrow in dorsal view, not fused with bulb, bulb yellow, same as long as cymbium, stout, tapering apically, embolus (em) curved prolaterally, flanked by distally narrowed, short skinny, bifid accessory structure (as) and short conductor (co) just below embolus (Figs 15A, B).

Female. Body length: 1.54 (Carapace, L: 0.54, W: 0.40 . Abdomen, L: $1.00, \mathrm{~W}: 0.50)$. In general similar to males. Sternum longer than wide, postepigastric scutum with posteriorly directed lateral apodemes (lap). Genitalia: Short, convoluted posterior genitalic tube (pgt) in between posterior spiracular groove (psg) and epigastric furrow (ef). Paddle-like sclerite (psc) present on the anterior genital area (Fig. 15D).

Distribution. Known only from the type locality (Fig. 21).

\section{Genus Silhouettella Benoit, 1979}

\section{Silhouettella saaristoi $\mathbf{n}$. sp.}

http://zoobank.org/08475B9A-BF90-4249-89C3-EB71E32A8E77 Figs $16 \mathrm{~A}-\mathrm{C}, 20 \mathrm{C}, \mathrm{E}$

Type material. Male holotype from Sri Lanka, Western Province, Colombo District, Labugama FR, 06 $51^{\circ} 17^{\prime \prime} \mathrm{N}$ $80^{\circ} 16^{\prime} 47^{\prime \prime}$ E, litter, 25 September 2014, leg. N. Athukorala (IFS_Oon_230) (ZFMK).

Other material examined: 1 male, 1 female; Western Province, Kalutara District, Gurulu Bedda, Kalugala FR, $06^{\circ} 26^{\prime} 35^{\prime \prime} \mathrm{N}, 80^{\circ} 14^{\prime} 52^{\prime \prime} \mathrm{E}, 40 \mathrm{~m}, 10$ January 2014, litter, leg. S. P. Benjamin, N. Athukorala (IFS_Oon_194, 277).

Diagnosis. Silhouettella saaristoi n. sp. resembles to Silhouettella snippy $\mathrm{n}$. sp. by the presence of curved, long embolus, but differ in having thin, long, curved embolus without a membranous flange (Fig. 20C). Females can be identified by short, narrow globular appendix (gap) with slight enlargement at base extends up to epigastric furrow (Fig. 20E).

Etymology. This species is named after Michael Saaristo (1938-2008) colleague and friend who worked extensively on the goblin spiders of the Seychelles.

Description. Male: Total length 1.22 (Carapace, L: 0.58, W: 0.42. Abdomen, L: 0.64, W: 0.56).

Coloration: carapace usually orange, sternum and mouthparts orange, abdominal scuta orange-yellow, legs pale orange, palps pale orange. Carapace elongated oval

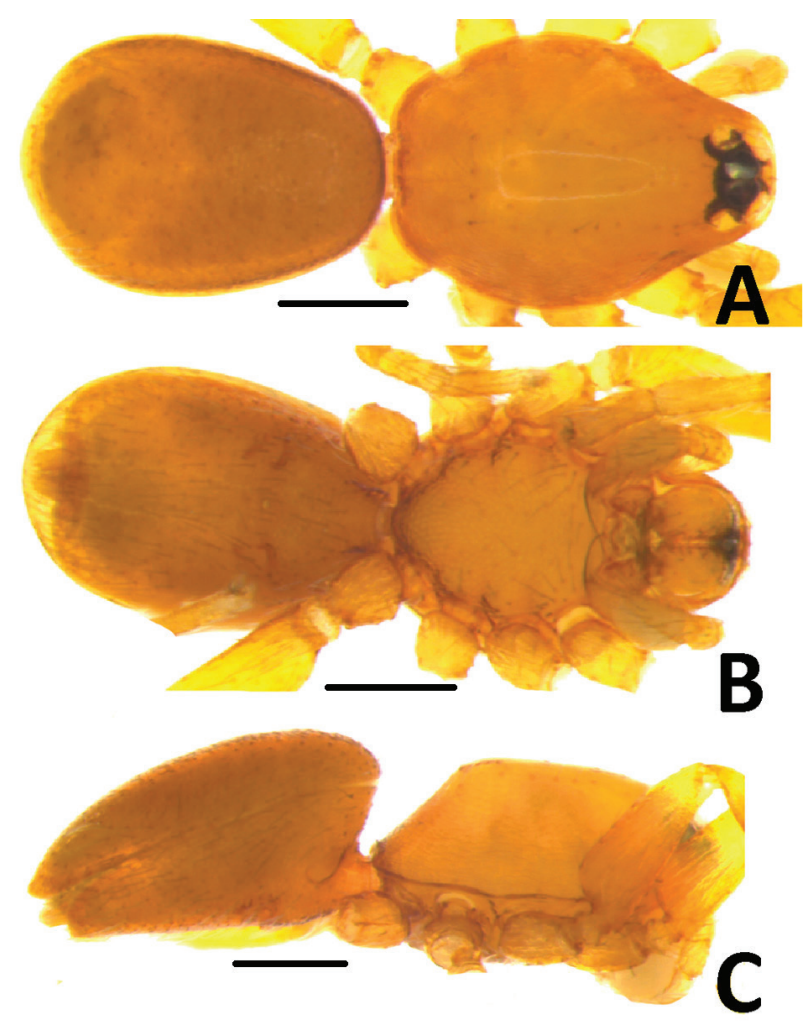

Figure 16. Silhouettella saaristoi n. sp., male from Labugama FR: A, habitus, dorsal view; B, same, ventral view; C, same, lateral view. Scale bars: $0.2 \mathrm{~mm}$. 
in dorsal view, anteriorly narrowed 0.5 times its maximum width (Fig. 16A), gradually elevated in lateral view (Fig. 16C), surface and sides smooth (Fig. 16C), posterior margin rounded. Clypeus straight in front view, slightly rebordered. Chelicerae straight, anterior face unmodified (Fig. 16A). Six eyes, well developed (Fig. 16A), ALE largest, circular, PME and PLE oval. Sternum longer than wide with scattered pits, radial furrows not visible in between coxae (Fig. 16B). Abdomen elongated, anteriorly narrowed (Fig. 16A), cover full length of abdomen, no soft tissue visible from above. Postepigastric scutum long, semicircular, covering nearly full length of abdominal length, fused to epigastric scutum, without posteriorly directed lateral apodemes (Fig. 16B). Posterior spiracles connected by groove (Fig. 16B), booklung covers small (Fig. 16B). Spinnerets scutum present as incomplete ring. Legs spines absent. Genitalia: Sperm pore small, elongated, situated at level of anterior spiracular groove (Fig. 16B). Palp of normal size, not strongly sclerotized, cymbium yellow, not fused with bulb, not extending beyond distal tip of bulb. Bulb yellow, rounded posteriorly. Embolus long and curve, visible as a thin-curved needle. Membranous flange just below embolus is absent (Fig. 20C).

Female: Total length 1.92 (Carapace, L: 0.78, W: 0.48. Abdomen, L: 1.14, W: 0.62). In general similar to males. Genitalia: Short, narrow globular appendix (gap) extends up to epigasrtic furrow with an anterior paddle like sclerite (psc) and a nail like process (na). Lateral protrusion at middle of gap ended with slight enlargement of the tube. Receptaculum wide and narrow (Fig. 20E).

Distribution. Labugama FR, Kalugala FR (Fig. 21).

\section{Silhouettella snippy n. sp.}

http://zoobank.org/9A4D8D51-4D20-425C-8520-C3DF873BA8E6 Figs 17A-H, 19A-C, 20A, D

Type material. Male holotype from Sri Lanka, Central Province, Matale District, NIFS Arboretum, 07 $51^{\prime} 34^{\prime \prime} \mathrm{N}$ $80^{\circ} 40^{\prime} 28^{\prime \prime} \mathrm{E}, 180 \mathrm{~m}$, litter, 23 July 2010, leg. S. Batuwita (IFS Oon 014) (ZFMK).

Other material examined: 1 male and 3 females; same locality, litter, 19 October 2015, leg. N. Athukorala et al. (IFS_Oon_322-325).

Diagnosis. Silhouettella snippy n. sp. can be diagnosed by the curved, long embolus with membranous flange just below embolus (Fig. 20A). Females can be identified by wide, semicircular receptaculum with short globular appendix (Fig. 20D).

Etymology. This species is a noun in apposition named after "Snippy" a brownie and one of the characters in the story "Billy's Little Boats" by Blyton in 1971.

Description. Male: Total length 1.52. (Carapace, L: 0.72, W: 0.41. Abdomen, L: 0.80, W: 0.44). Coloration: carapace orange, sternum and mouthparts orange, abdominal scuta orange-yellow, abdominal inter scutal region white and covered with setae, legs pale orange, palps pale orange. Carapace elongated oval in dorsal view, anteriorly narrowed 0.5 times its maximum width

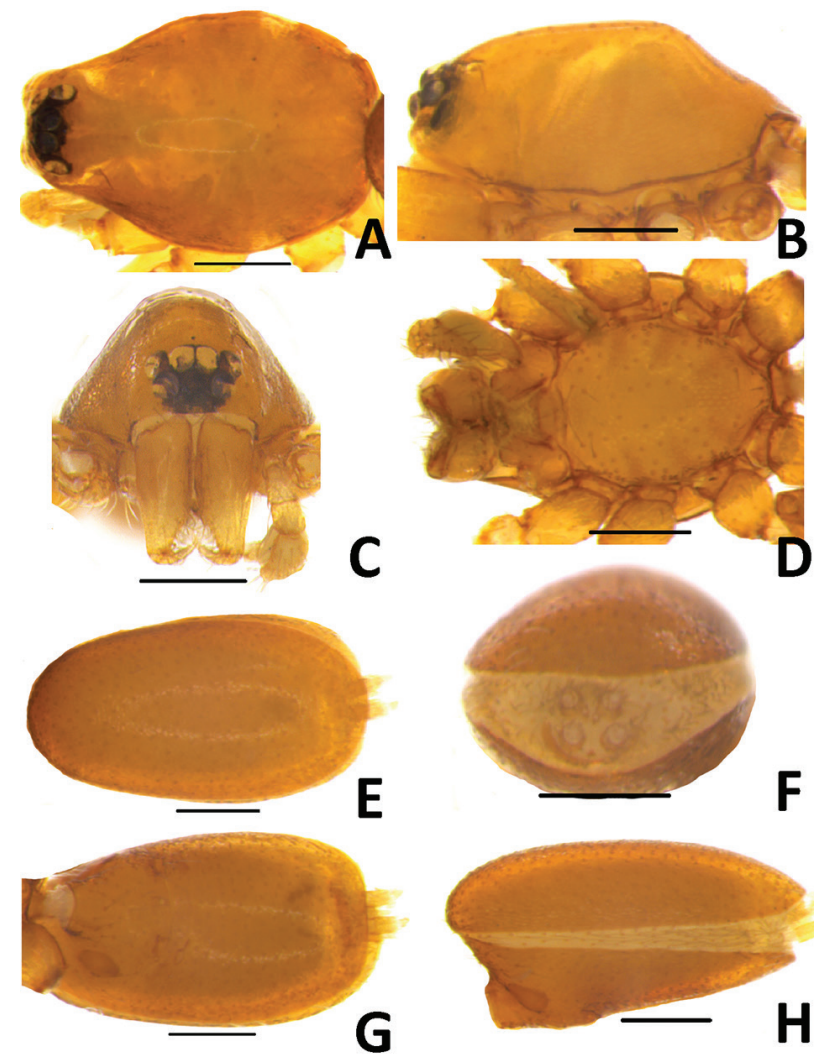

Figure 17. Silhouettella snippy n. sp., male from NIFS Arboretum: A, carapace, dorsal view; B, same, lateral view; C, same, anterior view; D, sternum, ventral view; E, abdomen, dorsal view; F, same, posterior view; G, same, ventral view; H, same, lateral view. Scale bars: $0.2 \mathrm{~mm}$.

(Fig. 17A), slightly elevated in lateral view (Fig. 17B), surface and sides smooth (Fig. 17B), posterior margin rounded. Clypeus slightly rebordered, straight in front view. Chelicerae straight, anterior face unmodified (Fig. 17C). Six eyes, well developed (Fig. 17C). Sternum longer than wide, radial furrows slightly visible in between coxae (Fig. 17D). Abdomen narrow, elongated (Fig. 17E), cover full length of abdomen, no soft tissue visible from above (Fig. 17E). Postepigastric scutum long, semicircular, covering nearly full length of abdominal length, fused to epigastric scutum, without posteriorly directed lateral apodemes (Fig. 17G). Posterior spiracles connected by groove (Fig. 17G), booklung covers large (Fig. 17G). Spinnerets scutum present as incomplete ring (Fig. 17F). Legs spines absent. Genitalia: Sperm pore small, situated at level of anterior spiracular groove (Fig. 17G). Palp of normal size, not strongly sclerotized, cymbium yellow, not fused with bulb, not extending beyond distal tip of bulb. Bulb yellow, rectangular-shaped, as long as cymbium. Embolus long, curved with a membranous flange just above conductor (Fig. 20A).

Female: Total length 2.00 (Carapace: L: 0.80, W: 0.66. Abdomen: L: 1.20, W: 0.88). In general similar to males (Fig. 19A). Genitalia: Receptaculum (re) semicircular, wider than long. Secretory sac (ssa) oval, globular appendix (gap) short, about 0.5 times the length of receptacu- 
lum with an anterior paddle like sclerite (psc) and lateral apodemes (lap) (Fig 20D).

Distribution. Known only from the type locality (Fig. 21).

\section{Silhouettella tiggy $\mathrm{n}$. sp.}

http://zoobank.org/90F71858-B93D-48B9-B12D-B02D70284AD3 Figs 18A-F, 19D-E, 20B

Type material. Male holotype from Sri Lanka, Central Province, Kandy District, Corbett's Gap, Knuckles range, $07^{\circ} 21^{\prime} 40^{\prime \prime} \mathrm{N} 80050^{\prime} 00^{\prime \prime} \mathrm{E}, 1360 \mathrm{~m}, 02$ November 2010, Hand collecting, leg. S. P. Benjamin, S. Batuwita (IFS Oon_034) (ZFMK).

Other material examined. 1 male; Sri Lanka, Central Province, Kandy District, Knuckles site 01, litter, 07 April 2015, leg. N. Athukorala (IFS_Oon_290); 1 female; Central Province, Kandy District, Dunumadalawa FR, $701 \mathrm{~m}, 07^{\circ} 16^{\prime} 38^{\prime \prime} \mathrm{N} 80^{\circ} 38^{\prime} 69^{\prime \prime} \mathrm{E}$, litter, 06 February 2017, leg. N. Athukorala et al. (IFS_Oon_449).

Diagnosis. Silhouettella tiggy n. sp. resembles S. usgutra Saaristo \& van Harten, 2002 by the rough, granulated carapace (Fig. 18A), but can be differentiated by the snout-shaped embolus-conductor complex (Fig. 20B).

Etymology. This species is a noun in apposition named after "Tiggy" a brownie and one of the characters in the story "Billy's Little Boats" by Blyton in 1971.

Description. Male: Total length 1.34 (Carapace, L: 0.58, W: 0.44. Abdomen, L: 0.76, W: 0.54). Coloration: carapace orange-brown, sternum and mouthparts orange, abdominal scuta orange-yellow, abdominal inter scutal region white and covered with setae, legs pale orange,
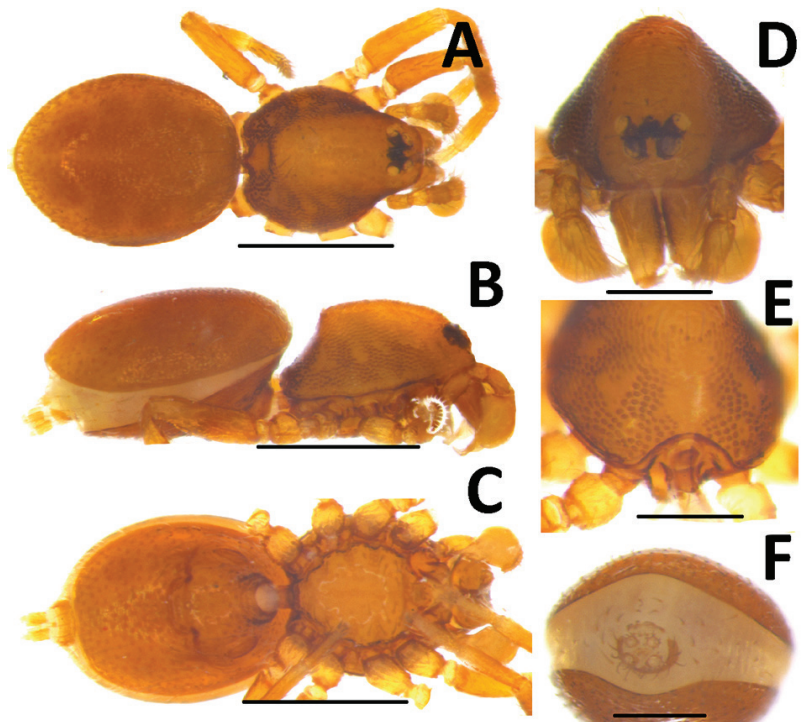

Figure 18. Silhouettella tiggy n. sp., male from Corbett's Gap: A, habitus, dorsal view; B, same, lateral view; C, same, ventral view; D, carapace, anterior view; E, same, posterior view; $\mathbf{F}$, abdomen, posterior view. Scale bars: $0.5 \mathrm{~mm}(\mathbf{A}-\mathbf{C}), 0.2 \mathrm{~mm}(\mathbf{D}-\mathbf{F})$. palps pale orange. Carapace broadly oval in dorsal view anteriorly narrowed 0.5 times its maximum width (Fig. 18A), strongly elevated in lateral view (Fig. 18B), surface and sides with the roughly granulated black serrations (Fig. 18B), posterior margin rounded (Fig. 18E). Clypeus slightly rebordered, straight in front view. Chelicerae straight, anterior face unmodified (Fig. 18D). Six eyes, well developed (Fig. 18D). Sternum as long as wide, radial furrows slightly visible in between coxae I-II, II-III, III-IV (Fig. 18C). Abdomen ovoid (Fig. 18A), dorsal scutum strongly sclerotized, pale orange, cover full length of
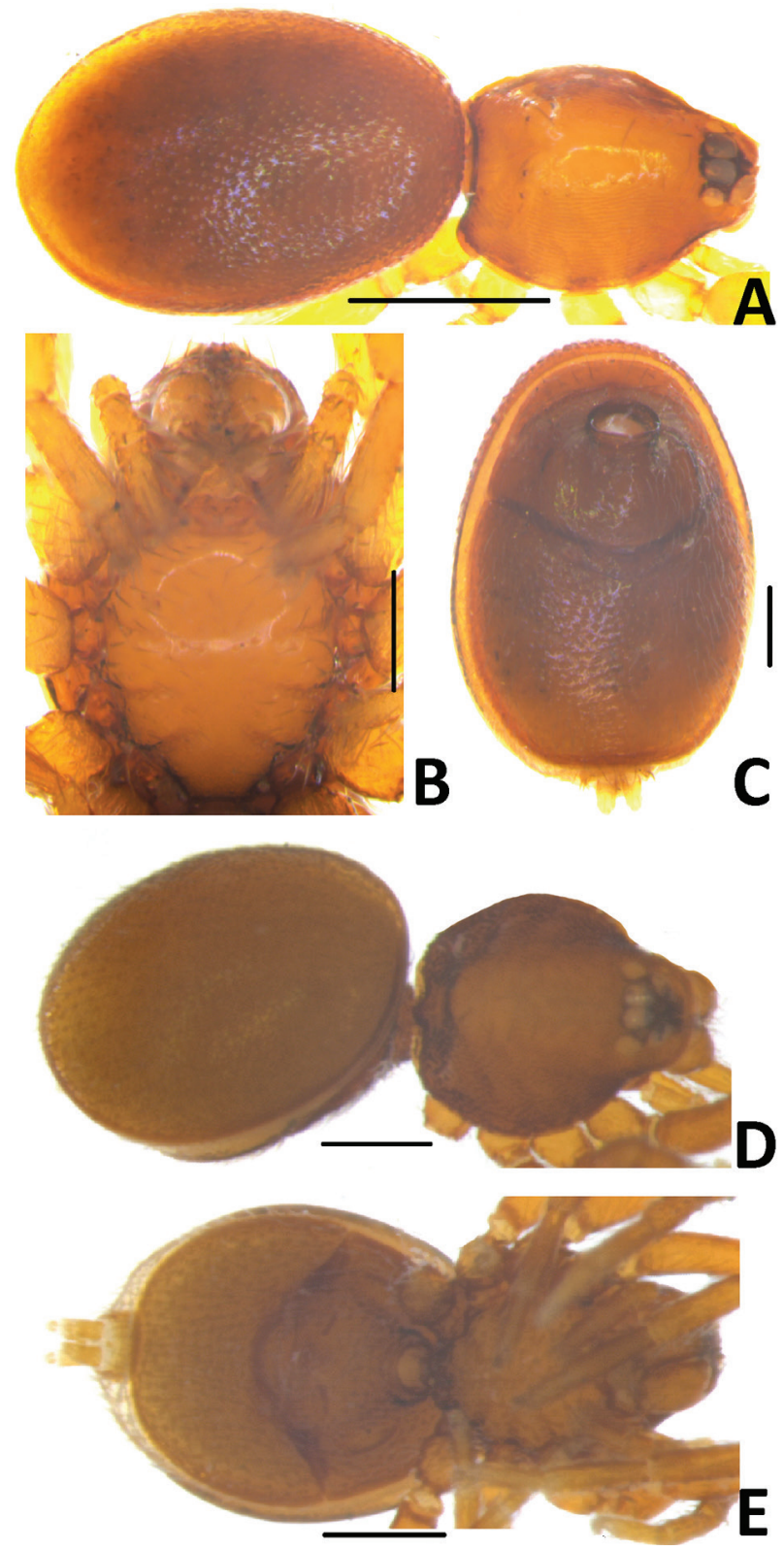

Figure 19. Silhouettella snippy n. sp., female from NIFS Arboretum (A-C), Silhouettella tiggy n. sp. female from Dunumadalawa FR (D-E): A, habitus, dorsal view; B, sternum, ventral view; C, abdomen, ventral view; $\mathbf{D}$, habitus, dorsal view; $\mathbf{E}$, habitus, ventral view. Scale bars: $0.5 \mathrm{~mm}(\mathbf{A}), 0.2 \mathrm{~mm}(\mathbf{B}-\mathbf{E})$. 


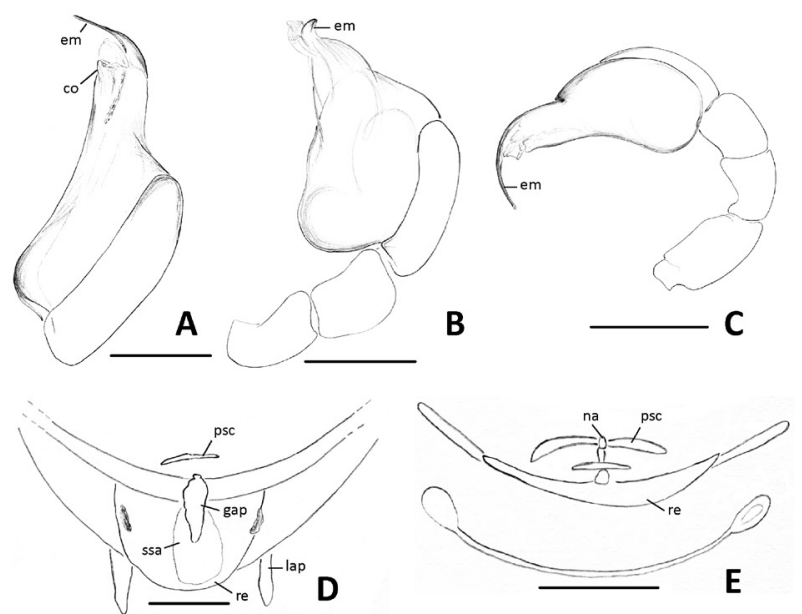

Figure 20. Silhouettella snippy n. sp. (A, D), Silhouettella tiggy n. sp. (B), Silhouettella saaristoi $\mathrm{n}$. sp. (C, E): A-C, male left palp, retrolateral view; $\mathbf{D}$, female epigastric region, dorsal view; E, same. Abbreviations: em, embolus; gap, globular appendix; lap, lateral apodemes na, nail like process; psc, paddle-like sclerite; re, receptaculum; ssa, secretory sac. Scale bars: $0.1 \mathrm{~mm}$.

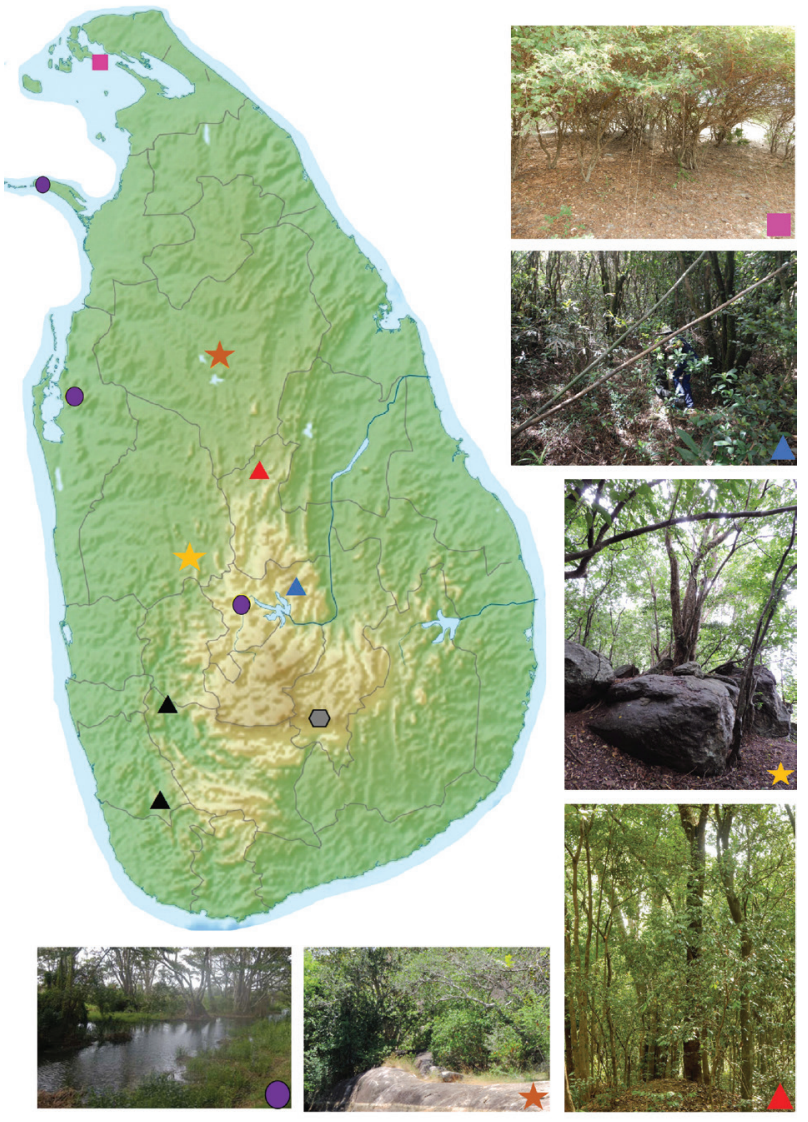

Figure 21. Distribution map of new species. Cavisternum bom n. sp.; Grymeus dharmapriyai n. sp.; Pelicinus snooky n. sp.; \ Pelicinus tumpy n. sp.; \ Silhouettella tiggy n. sp.; $\boldsymbol{\Delta}$ Silhouettella snippy n. sp.; $\boldsymbol{\Delta}$ Silhouettella saaristoi n. sp.; Ischnothyreus chippy n. sp. abdomen, no soft tissue visible from above (Fig. 18A). Postepigastric scutum long, semicircular, covering nearly full length of abdominal length, fused to epigastric scutum, without posteriorly directed lateral apodemes (Fig. 18C). Posterior spiracles connected by groove, booklung covers narrow (Fig. 18C). Spinnerets scutum present as incomplete ring (Fig. 18F). Legs spines absent. Genitalia: Sperm pore large, situated at level of anterior spiracular groove (Fig. 18C). Palp of normal size, not strongly sclerotized, cymbium yellow, not fused with bulb, not extending beyond distal tip of bulb. Bulb yellow, 1 to 1.5 times as long as cymbium, stout, spherical with snoutshaped embolus-conductor complex (Fig. 20B).

Female: Total length 1.32 (Carapace, L: 0.56, W: 0.46. Abdomen, L: 0.76, W: 0.58). Somatic morphology similar to the two males of Silhouettella tiggy n. sp. (Figs. 19D, E). Genitalia: Receptaculum length approximately $1 / 3$ of its width, between posterior spiracular groove and epigastric furrow. External genital structures on ventral scutum as in Fig. 19E.

Remarks. The association of this single female, collected from a locality further away from the type locality should be considered provisional.

Distribution. Corbett's Gap, Knuckles site 01, possibly in Dunumadalawa FR (Fig. 21).

\section{Discussion}

Here we report the discovery of nine new species of goblin spiders in six different genera: Cavisternum, Grymeus, Ischnothyreus, Opopaea, Pelicinus, and Silhouettella. The records of Cavisternum, Grymeus and Silhouettella are new to Sri Lanka.

Cavisternum only occurs in low rainfall sites with open woodland vegetation. Its habits generally fits the model for taxa that have adapted to arid environments in tropical northern Australia (Baehr et al., 2010). Endemism in Cavisternum appears to be high; most species have been recorded only from a single location. The same is true for Cavisternum bom n. sp. which is reported from Mandaitivu FR in Jaffna, a dry forest with an annual precipitation of $696 \mathrm{~mm}$ to $1125 \mathrm{~mm}$. This species is presently known only from its type locality. The relationship of C. bom n. sp. to the Australian species may be tested using DNA sequence data in future studies.

Three species of Grymeus were described previously from Australia (Harvey, 1987). Grymeus is not known from anywhere else in the world (World spider Catalog 2018) and is recognized by the presence of a pouch, setaceous book-lung covers and stout, blunt, carinate setae on dorsal scutum. Specimens of a single species, Grymeus dharmapriyai n. sp., were collected from three different localities in Sri Lanka (Fig. 21). This distribution of Grymeus dharmapriyai n. sp. differs from that of other species in being widespread, disjunct and occurring at 
both high and low elevations. Despite the unusual distribution, the males from the three sites are morphologically identical; characterized by their genital morphology, a dark red-brown, pear-shaped carapace and sternum with cell-shaped decorations.

Opopaea is a speciose, widely distributed genus of goblin spiders (World spider Catalog 2018). Male Opopaea are easily recognized by the combination of the enlarged palpal patella, that is attached sub-basally from the palpal femur and the fused bulbus and cymbium (Platnick \& Dupérré, 2009; Baehr et al., 2013b; Andriamalala \& Hormiga, 2013; Tong \& Li, 2015). Females are recognized by the triangular median depression on the posterior scutum, situated just behind the epigastric furrow (Saaristo, 2001). Previously, only a single species, $O$. mollis (Simon, 1907) has been reported from Sri Lanka. Epectris was synonymised with Opopaea by Baehr et al. (2013b); effectively transferring E. mollis to Opopaea. According to Simon's (1907) original description. “abdomine albido-lurido, cephalothorax sternumque laeviae tnitida, abdomen molle, haud scutatum", which according our translation means that the sternum is smooth and clean, abdomen is soft, no scutum and white-pale yellow. Therefore, this species might not belong to Opopaea or even a close relative, as its abdomen lacks a scutum (Platnick \& Dupérré, 2009; Simon, 1907). Thus, above we describe the first unequivocally identified species of the genus from the island. This species is unique in possessing four triangular-shaped spines on the posterior part of the carapace.

In stark contrast to most oonopids of the island, which are short-range endemics (Ranasinghe \& Benjamin, 2016a, b), O. spinosiscorona $\mathrm{n}$. sp. is widely distributed. It is even predictable that it might occur in neighboring India. Currently, Opopaea is not known from south Asia except for Bhutan (World spider Catalog 2018). However, the genus is well known from other parts of the world, mainly through work undertaken as part of the PBI Oonopidae project. Unfortunately, although many undescribed species from tropical Asia are know from museum collections, no revision of them is planned for the near future (Yvonne Kranz \& Norman I. Platnick pers. comm.).

Three new Silhouettella species and two Pelicinus species are newly reported from Sri Lanka. Both Pelicinus and Silhouettella belonged to the pelicinus-group. They have well-developed eyes that occupy most of the cephalic area and a wider female receptaculum associated with a globular apical process and lateral apodemes (Álvarez-Padilla et al., 2015). The female genitalia of $S$. saaristoi n. sp. (Fig. 20E) lack lateral apodemes and a large receptaculum, which are both characteristics of Silhouettella (Fig. 20D). However, we have no doubt that the single female is correctly assigned as the male and female are from Kalugala FR and were collected together (same date, time, collection event). However, a female collected from Dunumadalawa FR is only provisionally assigned to the male holotype of $S$. tiggy n. sp.

\section{Acknowledgments}

This study was funded by the National Institute of Fundamental Studies, Kandy. Special thanks to N. Athukorala for support in the field. We also thank S. Batuwita, H. Sandamali, C. Clayton, N. Kanesharatnam and I. Sandunika (all of NIFS) for collecting some of the described material. We are grateful to Dr. Norman Platnick, Dr. Y. Kranz-Baltensperger and Dr. Darrell Ubick for comments on various taxonomical problems related to the above descriptions. We are also grateful to Nadine Dupérré and an anonymous reviewer for reviewing this work. Thanks to S. Piyathissa for helping with the map. The Department of Wildlife Conservation and the Department of Forest Conservation of Sri Lanka provided permits for fieldwork.

\section{References}

Álvarez-Padilla F, Hormiga G (2008) A protocol for digesting internal soft tissues and mounting spiders for scanning electron microscopy. Journal of Arachnology 35: 538-542. https://doi.org/10.1636/ Sh06-55.1

Álvarez-Padilla F, Ubick D, Griswold CE (2015) Indian Ocean goblin spiders (Araneae, Oonopidae): four new species of pelicinoids from Madagascar, with a redescription of the type species Silhouettella curieusei Benoit, 1979. American Museum Novitates 3835: 1-31. https://doi.org/10.1206/3835.1

Andriamalala D, Hormiga G (2013) Systematics of the goblin spider genus Opopaea (Araneae, Oonopidae) in Magagascar. Bulletin of the American Museum of Natural History 380: 1-156. https://doi. org/10.1206/828.1

Azarkina G (2004) Two new species of the genus Aelurillus Simon, 1884 from Pakistan and Sri Lanka (Araneae: Salticidae). Bulletin of the British Arachnological Society 13: 49-52.

Baehr BC, Ubick D (2010) A review of the Asian goblin spider genus Camptoscaphiella (Araneae: Oonopidae). American Museum Novitates 3697: 1-65. https://doi.org/10.1206/3697.2

Baehr BC, Harvey MS, Smith HM (2010) The goblin spiders of the new endemic Australian genus Cavisternum (Araneae: Oonopidae). American Museum Novitates 3684: 1-40. https://doi. org/10.1206/667.1

Baehr BC (2011) Australian goblin spiders of the genus Opopaea Simon, part 1. The species of the IBISCA-Queensland project at Lamington National Park (Araneae: Oonopidae). Memoirs of the Queensland Museum, Nature 55: 413-437.

Baehr BC, Raven R, Whyte R (2013a) Biodiversity discovery program Bush Blitz yields a of goblin spider, Cavisternum attenboroughi (Araneae: Oonopidae), from the Northern Territory. Zootaxa 3616: 396-400. https://doi.org/10.11646/zootaxa.3616.4.8

Baehr BC, Harvey MS, Smith HM, Ott R (2013b) The goblin spider genus Opopaea in Australia and the Pacific islands (Araneae: Oonopidae). Memoirs of the Queensland Museum, Nature 58: 107-338.

Batuwita S, Benjamin SP (2014) An annotated checklist and a family key to the pseudoscorpion fauna. (Arachnida: Pseudoscorpiones) of Sri Lanka. Zootaxa, 3814 (1), 37-67. https://doi.org/10.11646/ zootaxa.3814.1.2 
Bayer S (2012) The lace-sheet-weavers - a long story (Araneae: Psechridae: Psechrus). Zootaxa 3379: 1-170.

Benjamin SP (2000) Epidius parvati sp. n., a new species of the genus Epidius from Sri Lanka (Araneae: Thomisidae). Bulletin of the British Arachnological Society 11: 284-288.

Benjamin SP (2001) The genus Oxytate L. Koch 1878 from Sri Lanka, with description of Oxytate taprobane sp. n. (Araneae: Thomisidae). Journal of the Society for Asian Natural History 5: 153-158.

Benjamin SP, Jocqué R (2000) Two new species of the genus Suffasia from Sri Lanka (Araneae: Zodariidae). Revue Suisse de Zoologie 107: 97-106. https://doi.org/10.5962/bhl.part.80120

Benjamin SP (2004) Taxonomic revision and phylogenetic hypothesis for the jumping spider subfamily Ballinae (Araneae, Salticidae). Zoological Journal of the Linnean Society 142: 1-82. https://doi. org/10.1111/j.1096-3642.2004.00123.x

Benjamin SP (2006) The male of Marengo nitida with the description of $M$. rattotensis new species from Sri Lanka (Araneae: Salticidae). Zootaxa 1326: 25-36.

Benjamin SP (2010) Revision and cladistic analysis of the jumping spider genus Onomastus (Araneae: Salticidae). Zoological Journal of the Linnean Society 159: 711-745. https://doi.org/10.1111/j.10963642.2009.00580.x

Benjamin SP (2015) Model mimics: antlike jumping spiders of the genus Myrmarachne from Sri Lanka. Journal of Natural History 49(43-44): 2609-2666. https://doi.org/10.1080/00222933.2015.1034209

Benjamin SP, Kanesharatnam N (2016) Description of three new species of the tropical Asian jumping spider genus Onomastus Simon, 1900 from high altitude cloud forests of Sri Lanka (Araneae: Salticidae). Zootaxa 4205: 431-453. https://doi.org/10.11646/zootaxa.4205.5.2 Blyton E (1947) The Holiday Book. Sampson Low, London, 388 pp. Blyton E (1971) Toyland Tales. Purnell \& Sons, London, 156 pp.

Dong TT, Zheng G, Yao ZY, Li SQ (2016) Fifteen new species of the spider genus Pholcus (Araneae: Pholcidae) from Southeast Asia. Zootaxa 4136: 201-246. https://doi.org/10.11646/zootaxa.4136.2.1

Dunlop JA, Jekel D (2009) Nomenclatural notes on fossil spiders. Bulletin of the British Arachnological Society 14: 357-360. https://doi. org/10.13156/100.014.0903

Grismado CJ, Deeleman C, Baehr B (2011) The goblin spider genus Aprusia Simon, 1893 (Araneae: Oonopidae). American Museum Novitates 3706: 1-21. https://doi.org/10.1206/3706.2

Eichenberger B, Kranz-Baltensperger Y, Ott R, Graber W, Nentwig W, Kropf C (2012) Morphology of new Indian/ Indonesian Gamasomorpha and Xestaspis species (Araneae: Oonopidae). Zootaxa 3160, 1-68.

Harvey MS, Baehr BC (2013) Two new species of the goblin spider genus Cavisternum form tropical Australia (Araneae: Oonopidae). Memoirs of the Queensland Museum, Nature 58: 359-366.

Harvey MS (1987) Grymeus, a new genus of pouched oonopid spider from Australia (Chelicerata: Araneae). Memoirs of the Museum of Victoria 48: 123-130. https://doi.org/10.24199/j. mmv.1987.48.25

Huber BA (2005) High species diversity, male-female coevolution, and metaphyly in southeast Asian pholcid spiders: the case of Belisana Thorell 1898 (Araneae, Pholcidae). Zoologica 155: 1-126.

Huber BA (2011) Revision and cladistic analysis of Pholcus and closely related taxa (Araneae, Pholcidae). Bonner Zoologische Monographien 58: 1-509.

Huber BA, Benjamin SP (2005) The pholcid spiders from Sri Lanka: redescription of Pholcus ceylonicus and description of a new ge- nus (Araneae: Pholcidae). Journal of Natural History 39: 33053319. https://doi.org/10.1080/00222930500145123

Jäger P (2003) Rhitymna Simon 1897: an Asian, not an African spider genus. Generic limits and descriptions of new species (Arachnida, Araneae, Sparassidae). Senckenbergiana Biologica 82: 99-125.

Kanesharatnam N, Benjamin SP (2016) Three new generic records and descriptions of four new species of jumping spiders (Araneae, Salticidae) from Sri Lanka. European Journal of Taxonomy 228: 1-23.

Kim JP, Ye SH, Oh JH (2013) A new species of the genus Saitis Simon, 1876 (Araneae: Salticidae) from Sri Lanka. Korean Arachnology 29: 251-262.

Kim JP, Ye SH (2014) A new species of the genus Ctenus Walckenaer, 1805 (Araneae: Ctenidae) from Sri Lanka. Korean Arachnology 30(1): 61-70.

Nanayakkara RP, Kirk PJ, Dayananda SK, Ganehiarachchi GASM, Vishvanath N, Kusuminda TGT (2012) A new species of tiger spider, genus Poecilotheria, from northern Sri Lanka. Journal of the British Tarantula Society 28: 6-15.

Ott R, Harvey MS (2008) A new species of Pelicinus from Barrow Island, Western Australia (Araneae: Oonopidae). Arthropoda Selecta 17: $81-85$.

Platnick NI, Dupérré N (2009) The goblin spider genera Opopaea and Epectris (Araneae, Oonopidae) in the New World. American Museum Novitates 3649, 1-43. https://doi.org/10.1206/664.1

Platnick NI, Dupérré N, Ott R, Kranz-Baltensperger Y (2011) The goblin spider genus Brignolia (Araneae, Oonopidae). Bulletin of the American Museum of Natural History 349: 1-131. https://doi. org/10.1206/743.1

Platnick NI, Dupérré N, Ott R, Baehr BC, Kranz-Baltensperger Y (2012) The goblin spider genus Pelicinus (Araneae, Oonopidae), Part 1. American Museum Novitates 3741: 1-43. https://doi. org/10.1206/3741.2

Polotow D, Griswold C (2017) Cleaning old cabinets: revealing the taxonomy of Sri Lankan wolf spiders (Araneae, Udubidae and Zoropsidae).

Ranasinghe UGSL, Benjamin SP (2016a) A review of Sri Lankan Brignolia including the description of four new species (Araneae: Oonopidae). Zootaxa 4144(4): 451-476. https://doi.org/10.11646/ zootaxa.4144.4.1

Ranasinghe UGSL, Benjamin SP (2016b) The goblin spider genus Xestaspis in Sri Lanka (Araneae: Oonopidae). Zootaxa 4189(1): 6080. https://doi.org/10.11646/zootaxa.4189.1.2

Ranasinghe UGSL, Benjamin SP (2016c) New records of Pelicinus and Xyphinus from Sri Lanka (Araneae: Oonopidae). Indian Journal of Arachnology 5: 71-78. https://doi.org/10.1080/00222933.2 018.1444803

Ranasinghe UGSL, Benjamin SP (2018) Three new species of Aprusia (Araneae: Oonopidae) from Sri Lanka with a phylogenetic analysis of the genus. Journal of Natural History 52 (11-12): 713-738.

Ranasinghe S (2017) Dwarf Hunters in Tropical Forests, The Newsletter British Arachnological Society 140: 7-8.

Saaristo MI (2001) Dwarf hunting spiders or Oonopidae (Arachnida, Araneae) of the Seychelles. Insect Systematics and Evolution 32, 307-358.

Saaristo MI (2007) The oonopid spiders (Aranei: Oonopidae) of Israel. Arthropoda Selecta 15: 119-140. https://doi. org/10.1163/187631201X00236

Saaristo MI, Harten A van (2002) The oonopid spiders (Arachnida: Araneae: Oonopidae) of Socotra, Yemen. Fauna of Arabia 19, 311-319. 
Smith AM (2004) A new species of the arboreal theraphosid, genus Poecilotheria, from southern India (Araneae, Mygalomorphae, Theraphosidae) with notes on its conservation status. Journal of the British Tarantula Society 19: 48-61.

Simon E (1890) Etudes arachnologiques. 22e Mémoire. XXXIV. Etude sur les arachnides de 1'Yemen. Annales de la Société Entomologique de France 10(6):77-124.

Simon E (1892) On the spiders of the island of St. Vincent. Part 1. Proceedings of the Zoological Society of London 59(4, for 1891): 549575, P1. XLII.
Simon E (1893a) Histoire naturelle das araignées. Paris 1, 257-488.

Simon E (1907) Etude sur les araignées de la sous-section des Haplogynes. Annales de la Société Entomologique de Belgique 51: 246264. https://doi.org/10.5962/bhl.part.1584

Tong YF, Li SQ (2015) Six new species of the genus Opopaea Simon, 1891 from Xishuangbanna Rainforest, southwestern China (Araneae: Oonopidae). Zootaxa 3931(1): 41-62. https://doi.org/10.11646/ zootaxa.3931.1.3

World Spider Catalog (2018) World Spider Catalog. Natural History Museum Bern, Available from: http://wsc.nmbe.ch [version 19, accessed 21 March 2018] 